\title{
FH535 inhibited metastasis and growth of pancreatic cancer cells
}

This article was published in the following Dove Press journal:

OncoTargets and Therapy

6 July 2015

Number of times this article has been viewed

\author{
Meng-Yao Wu ${ }^{1, *}$ \\ Rong-Rui Liangl,* \\ Kai Chen' \\ Meng Shen' \\ Ya-Li Tian ${ }^{1,2}$ \\ Dao-Ming Li' \\ Wei-Ming Duan' \\ Qi Gui' \\ Fei-Ran Gong ${ }^{3}$ \\ Lian Lian ${ }^{1,2}$ \\ Wei $\mathrm{Li}^{1,6}$ \\ Min Tao ${ }^{1,4-6}$ \\ 'Department of Oncology, The First \\ Affiliated Hospital of Soochow \\ University, ${ }^{2}$ Department of Oncology, \\ Suzhou Xiangcheng People's Hospital, \\ ${ }^{3}$ Department of Hematology, The \\ First Affiliated Hospital of Soochow \\ University, ${ }^{4}$ Jiangsu Institute of \\ Clinical Immunology, The First \\ Affiliated Hospital of Soochow \\ University, ${ }^{5}$ Institute of Medical \\ Biotechnology, Soochow University, \\ Suzhou, ${ }^{6}$ PREMED Key Laboratory \\ for Precision Medicine, Soochow \\ University, Suzhou, People's Republic \\ of China \\ *These authors contributed equally \\ to this work
}

Correspondence: Wei Li; Min Tao Department of Oncology, The First Affiliated Hospital of Soochow University, Suzhou 215006, People's Republic of China

Tel +86 $67780315 ;+8667780310$

$\mathrm{Fax}+865126238798$

Email liweil0@suda.edu.cn; mtao@medmail.com.cn

\begin{abstract}
FH535 is a small-molecule inhibitor of the Wnt/ $\beta$-catenin signaling pathway, which a substantial body of evidence has proven is activated in various cancers, including pancreatic cancer. Activation of the Wnt/ $\beta$-catenin pathway plays an important role in tumor progression and metastasis. We investigated the inhibitory effect of FH535 on the metastasis and growth of pancreatic cancer cells. Western blotting and luciferase reporter gene assay indicated that FH535 markedly inhibited Wnt/ $\beta$-catenin pathway viability in pancreatic cancer cells. In vitro wound healing, invasion, and adhesion assays revealed that FH535 significantly inhibited pancreatic cancer cell metastasis. We also observed the inhibitory effect of FH535 on pancreatic cancer cell growth via the tetrazolium and plate clone formation assays. Microarray analyses suggested that changes in the expression of multiple genes could be involved in the anti-cancer effect of FH535 on pancreatic cancer cells. Our results indicate for the first time that FH535 inhibits pancreatic cancer cell metastasis and growth, providing new insight into therapy of pancreatic cancer.
\end{abstract}

Keywords: pancreatic cancer, FH535, $\beta$-catenin, metastasis, growth

\section{Introduction}

Pancreatic cancer is one of the most aggressive human malignancies worldwide. Despite improvements in surgical and chemotherapeutic approaches over the past decades, the prognosis of pancreatic cancer remains dismal; the average overall 5 -year survival rate is $<5 \%{ }^{1}$ The reasons for this are the challenges associated with diagnosis, which tends to be late and uncertain; more importantly, therapeutic options are limited. Even with early diagnosis and surgical resection with curative intention, nearly all patients develop local recurrence or distant metastases following surgery and eventually succumb to the debilitating effects of metastatic growth. ${ }^{2,3}$ Conventional chemotherapy is rarely curative for metastatic pancreatic cancer. In recent years, there have been important advances in the organization of care for patients with pancreatic cancer; these advances have also resulted in more focused studies on surgical, oncological, and immunological treatment.

The Wnt/ $\beta$-catenin pathway is a genetically conserved signaling pathway associated with a variety of human conditions such as birth defects and tumors. Abnormal Wnt/ $\beta$-catenin pathway activation is closely related to the development of many cancers. ${ }^{4,5}$ An increasing amount of evidence demonstrates that both the $\beta$-catenin-dependent (canonical) and $\beta$-catenin-independent (non-canonical) Wnt signaling pathways play a key role in regulating pathological processes by facilitating tumor growth, migration, and invasion. In canonical Wnt signaling, glycogen synthase kinase-3 $\beta$ (GSK-3 $\beta$ ) phosphorylates $\beta$-catenin at certain key residues, leading to its ubiquitination and subsequent degradation. ${ }^{5,6}$ Non-phosphorylated $\beta$-catenin accumulates in the cytoplasm, 
and pathway activation leads to nuclear accumulation of $\beta$-catenin and interaction with T-cell factor (TCF) transcription factors, subsequently stimulating the downstream target genes, which include the genes participating in cell metastasis and proliferation. ${ }^{7,8}$

Abnormal Wnt/ $\beta$-catenin pathway activation plays an important role in human pancreatic cancer, where it causes extracellular matrix degradation and uncontrolled cell proliferation and differentiation. ${ }^{9}$ Recent studies have demonstrated that FH535 is a synthetic inhibitor of the canonical Wnt signaling pathway; it inhibits the growth of colon, lung, breast, and hepatocellular carcinoma lines, ${ }^{10,11}$ suggesting that small-molecule targeting of the Wnt/ $\beta$-catenin pathway could be a promising therapeutic approach for cancers in which this pathway is activated.

In this study, we investigated the anti-cancer effect of FH535 on pancreatic cancer and explored the mechanisms underlying the effect, providing a rationale for further development of FH535 as a promising therapeutic agent for treating pancreatic cancer.

\section{Materials and methods}

\section{Cell cultures and reagents}

The human pancreatic cancer cell lines PANC-1 and BxPC-3 were purchased from American Type Culture Collection (ATCC) (Manassas, VA, USA). The cells were maintained in Dulbecco's Modified Eagle's Medium (DMEM; Thermo Fisher Scientific, Waltham, MA, USA) supplemented with $10 \%$ fetal calf serum (FCS), $100 \mathrm{U} / \mathrm{mL}$ penicillin, and $100 \mu \mathrm{g} / \mathrm{mL}$ streptomycin (Thermo Fisher Scientific) at $37^{\circ} \mathrm{C}$ in a $5 \%$ $\mathrm{CO}_{2}$ incubator under a humidified atmosphere; the cells were passaged every 2-3 days for exponential growth. FH535 was purchased from EMD Millipore (Billerica, MA, USA).

\section{Western blotting}

Total protein was extracted using a lysis buffer $(50 \mathrm{mM}$ Tris$\mathrm{HCl}$ [pH 7.4], $150 \mathrm{mM} \mathrm{NaCl}, 1 \%$ Triton X-100, 0.1\% sodium dodecyl sulfate [SDS], $1 \mathrm{mM}$ EDTA) supplemented with a protease inhibitor cocktail kit and a phosphatase inhibitor cocktail kit (Hoffman-La Roche Ltd., Basel, Switzerland). The protein extracts were loaded, size-fractionated by SDSpolyacrylamide gel electrophoresis, and transferred to polyvinylidene difluoride membranes (Bio-Rad Laboratories Inc., Hercules, CA, USA). After blocking, the membranes were incubated with the primary antibodies mouse anti- $\beta$-catenin (Santa Cruz Biotechnology Inc., Dallas, TX, USA) and rabbit anti- $\beta$-actin (Proteintech Group Inc., Chicago, IL, USA) at $4^{\circ} \mathrm{C}$ overnight. Protein expression was determined using horseradish peroxidase-conjugated anti-mouse or anti-rabbit secondary antibodies, followed by detection using enhanced chemiluminescence (EMD Millipore). Band intensity was visualized using a JS-1035 image analysis scanning system (Shanghai Peiqing Science \& Technology, Co., Ltd., Shanghai, People's Republic of China).

\section{Luciferase reporter assay}

$\beta$-catenin is a dominant factor in the Wnt/ $\beta$-catenin/TCF signaling pathway, which regulates gene transcription by binding $\beta$-catenin and TCF. The activity of this final step in the pathway can be precisely measured using a luciferase reporter construct. The reporter plasmid pTOPFLASH (TCF reporter plasmid; EMD Millipore) contains two sets (the second set is in the reverse orientation) of three copies of the TCF binding site (wild-type) upstream of the thymidine kinase minimal promoter and luciferase open reading frame. The internal control plasmid pRL-SV40 (Promega Corporation, Fitchburg, WI, USA) contains the Renilla luciferase gene. Cells were transiently cotransfected with pTOPFLASH plasmid (500 ng/well) and pRL-SV40 plasmid (100 ng/well) for 6 hours using Lipofectamine 2000 (Thermo Fisher Scientific) according to the manufacturer's protocol. Then, the medium was renewed and FH535 was added. After 24 hours of treatment, cell lysates were subjected to the dual luciferase reporter assay according to the manufacturer's recommendations; luciferase activity was measured using a luminometer (Turner Designs, Sunnyvale, CA, USA). The results are expressed as relative luciferase activity, ie, the ratio of firefly luciferase activity over Renilla luciferase activity.

\section{Wound healing assay}

Cells $\left(1 \times 10^{4} /\right.$ well $)$ were seeded in 96-well plates and grown to confluence. The monolayer culture was artificially scrape wounded with a sterile micropipette tip to create a denuded zone of constant width. Each well was washed with phosphate-buffered saline twice to remove the detached cells before FH535 treatment. Cell migration to the wounded region was observed using an XDS-1B inverted microscope (MIC Optical and Electrical Instrument, Chongqing, People's Republic of China) and photographed ( $\times 40$ magnification). Images were captured at 0,8 , and 12 hours to monitor the wound healing process. The wound areas were measured using ImageJ (NIH, Bethesda, MA, USA).

\section{Transwell invasion assay}

We used a 24-well Transwell plate with an $8 \mu \mathrm{m}$ pore size polycarbonate filter membrane (Corning Incorporated, Corning, NY, USA). Cells $\left(1 \times 10^{5}\right)$ in $100 \mu \mathrm{L}$ serum-free DMEM were added to the Matrigel-coated top chamber (BD Biosciences, San Jose, CA, USA); the bottom chamber contained 
DMEM with $10 \%$ FCS. The cells were incubated for 24 hours; cells that had invaded through the Matrigel-coated membrane were fixed and stained with crystal violet and counted under a light microscope in five random fields in a blinded fashion.

\section{Adhesion assay}

Cells were resuspended in complete medium and seeded in 24-well plates at $1 \times 10^{4}$ cells $/ \mathrm{mL}$. After 5 -hour incubation, the unattached cells were removed to another well. The attached and unattached cells were evaluated using the 3-[4,5dimethylthiazol-2-yl] 2,5-diphenyltetrazolium bromide (MTT) assay. The adhesion rate was calculated as follows: (absorbance of attached cells/[absorbance of attached cells + absorbance of unattached cells]) $\times 100 \%$.

\section{MTT assay}

Cell growth was evaluated using the MTT assay. Cells $\left(5 \times 10^{4} /\right.$ well) were seeded in 24-well tissue culture plates. Blank control was treated with DMSO. After FH535 treatment, MTT (SigmaAldrich Co., St Louis, MO, USA) was added to each well (final concentration, $0.5 \mathrm{mg} / \mathrm{mL}$ ), followed by 4-hour incubation at $37^{\circ} \mathrm{C}$. The medium was removed, and $800 \mu \mathrm{L}$ of dimethyl sulfoxide was added to each well. The absorbance of the mixture was measured at $490 \mathrm{~nm}$ using a microplate enzyme-linked immunosorbent assay reader (Bio-Rad Laboratories Inc.). The relative cell viability was calculated as follows: relative cell viability $=($ mean experimental absorbance $/$ mean control absorbance) $\times 100 \%$.

\section{Plate clone formation assay}

Cells (200/well) were seeded in 24-well plates and treated after 12 hours. After 15 days, the cells were stained with $1 \%$ methylrosanilinium chloride, and the number of visible colonies was counted. The relative clone formation ability was calculated as follows: (mean experimental clone number/ mean control clone number) $\times 100 \%$.

\section{Cell cycle analysis}

Before treatment, the cells were serum starved for 24 hours to synchronize the cell cycle. Then, FCS was added to the cells, followed by various concentrations of FH535. Following 24 hours of FH535 treatment, the cells were fixed in $80 \%$ cooled ethanol and incubated with $0.5 \%$ Triton $\mathrm{X}-100$ solution containing $1 \mathrm{mg} / \mathrm{mL}$ RNase A at $37^{\circ} \mathrm{C}$ for 30 minutes. Next, propidium iodide (Sigma-Aldrich Co.) was added to the wells (final concentration, $50 \mu \mathrm{g} / \mathrm{mL}$ ), followed by 30 -minute incubation in the dark. Cellular DNA content was analyzed using a fluorescence-activated cell sorter (Becton Dickinson, Franklin Lakes, NJ, USA). Data were processed using ModFit LT software (Verity Software House, Topsham, ME, USA).

\section{Microarray assay}

Sample preparation and processing were performed as described in the GeneChip Expression Analysis Manual (Agilent Technologies, Santa Clara, CA, USA). Differentially expressed genes were screened using Agilent 44K human whole-genome oligonucleotide microarrays. The selection criterion was greater than twofold difference in expression (difference in upregulated expression was greater than twofold; difference in downregulated expression was less than 0.5-fold). Hierarchical clustering of samples was performed using an average linkage algorithm using TIGR MultiExperiment Viewer (The Institute for Genomic Research, Rockville, MD, USA).

\section{Statistical analysis}

Each experiment was performed in at least triplicate. Results are expressed as the mean \pm standard deviation. Statistical analysis was performed using an unpaired Student's $t$-test. $P<0.05$ was considered significant.

\section{Results \\ FH535 inhibited the $\beta$-catenin pathway in pancreatic cancer cells}

Treatment with $20 \mu \mathrm{M}$ FH535 ${ }^{12}$ did not affect nuclear or total $\beta$-catenin expression in the BxPC-3 cells, but downregulated nuclear and total $\beta$-catenin in the PANC-1 cells (Figure 1A). The luciferase reporter assay confirmed that FH535 suppressed TCF-dependent transcription, which may have led to dysregulation of the genes downstream of the $\beta$-catenin pathway (Figure 1B). To verify this, we performed microarray analyses to determine the mRNA expression changes in 138 genes downstream of the $\beta$-catenin pathway using Agilent 44K human whole-genome oligonucleotide microarrays (http://www.stanford.edu/group/nusselab/cgi-bin/wnt/ target genes); $20 \mu \mathrm{M}$ FH535 upregulated or downregulated multiple genes (Figure 1C, Table 1).

\section{FH535 inhibited pancreatic cancer cell migration}

In all, $20 \mu \mathrm{M}$ FH535 inhibited pancreatic cancer cell migration in a time-dependent manner (Figure 2A). To investigate the mechanisms involved, we analyzed the microarray data to illustrate the expression of genes participating in focal adhesion (Figure 2B, Table 2), ${ }^{13,14}$ adhesion junctions (Figure 2C, Table 3), ${ }^{15-17}$ tight junctions (Figure 2D, Table 4), ${ }^{18-23}$ and cell motility (Figure 2E, Table 5). ${ }^{24-27}$ 


\section{FH535 inhibited pancreatic cancer cell invasion}

The Matrigel invasion assay revealed that FH535-treated cells had significantly decreased invasive capacity as compared with the control cells (Figure 3A), supporting the premise that FH535 inhibits pancreatic cancer cell invasion. Moreover, FH535 inhibited the adhesion ability of pancreatic cancer cells dose-dependently (Figure 3C). We also analyzed the microarray data to explore the changes in the expression of genes involved in the in vitro invasion process, including extracellular matrix degradation (Figure 3B, Table 6), cell adhesion (Figure 3D, Table 7), ${ }^{28,29}$ and epithelial-mesenchymal transition (EMT) (Figure 3E, Table 8). ${ }^{30-33}$

\section{FH535 inhibited pancreatic cancer cell growth}

Using MTT assay, we evaluated the inhibitory effect of FH535 on pancreatic cancer cell line growth. The proliferation of PANC-1 and BxPC-3 cells cultured for up to 48 hours with FH535 was significantly inhibited time-dependently and dose-dependently as compared to the control cells (Figure 4A). The clone formation assays confirmed the dose-dependent inhibitory effect of FH535 on pancreatic cancer cell growth (Figure 4B). We performed cell cycle analysis to confirm the antimitogenic effect of FH535. FH535 induced G2/M accumulation and decreased the cell population in the G0/G1 and $\mathrm{S}$ phases dose-dependently (Figure 4C). The expression profile of the cell cycle-related genes obtained from microarray analyses was analyzed (Figure $4 \mathrm{D}$, Table 9). ${ }^{34}$

\section{Discussion}

It is widely acknowledged that the prognosis of pancreatic cancer is very poor. The canonical Wnt/ $\beta$-catenin signaling pathway plays a key role in tumor development and dissemination. Classical Wnt signaling pathway causes accumulation of $\beta$-catenin in cytoplasm in complex with the transcription factor TCF/LEF that regulates target gene expression. ${ }^{9,35}$ Dysregulation of $\mathrm{Wnt} / \beta$-catenin signaling and altered transcription of $\beta$-catenin/TCF-regulated genes are found in many cancers, ${ }^{36}$ including pancreatic cancer ${ }^{37}$ In this regard, we focused on characterizing the mechanisms of the anti-tumor effect of FH535 on pancreatic cancer cells.

Western blotting revealed that FH535 did not affect $\beta$-catenin expression in BxPC-3 cells. Interestingly, FH535

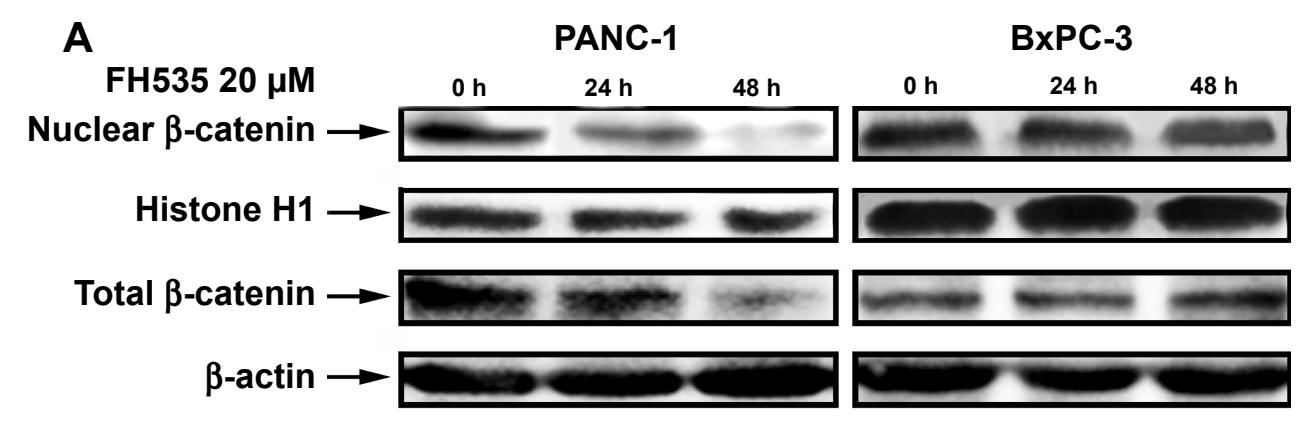

B
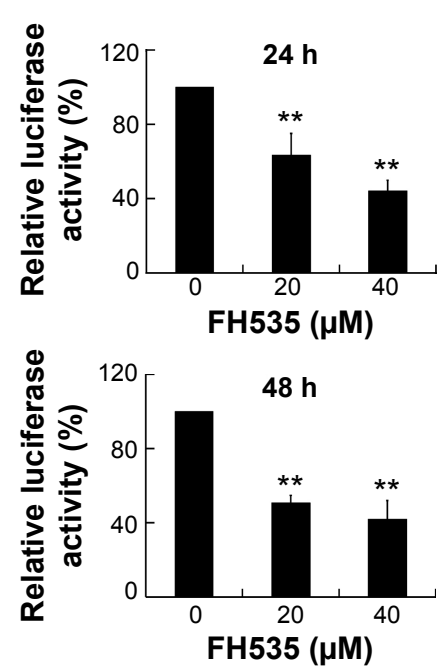

BxPC-3
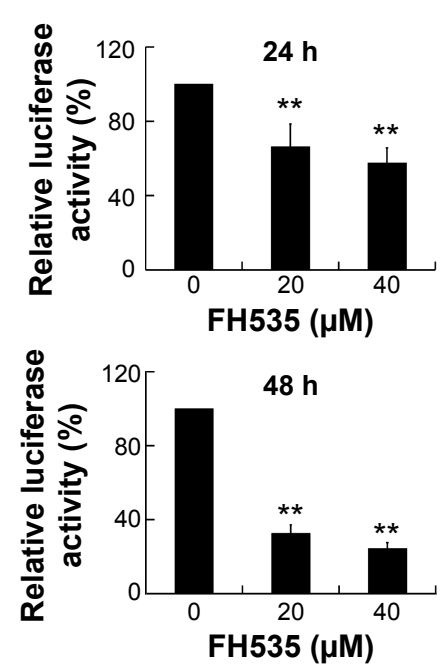

Figure I (Continued) 
C

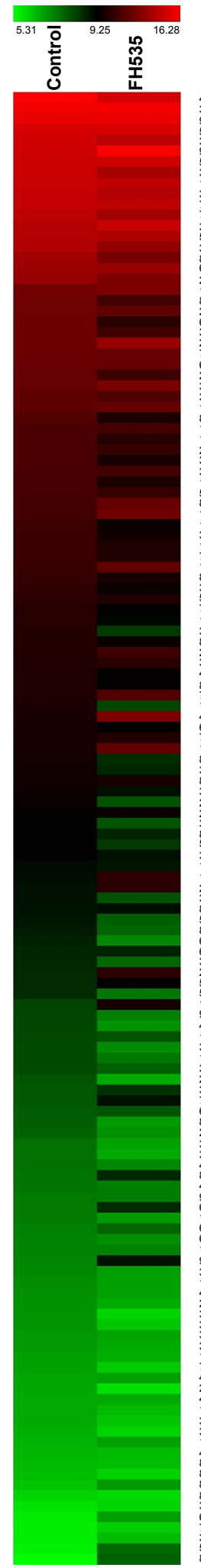

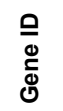

$0^{x^{5}}$
$M Y C$

CD44

WISP2

FOSL1

SNA11

KRT73

GJA1

$D K K 3$

CCND3

KRT83

CEBPD

EGFR

CDX4

ISLR

CTGF

KRT85

TLE1

CDX1

KRT8

TLAM1

EGR1

MET

AHR

CACNA1G

KRT15

KRT23

KRT39

DAB2

ETS2

KRT31

RET

UBXN6

STRA6

UBXN4

KRT76

UBXN2B

LRP1

KRTS

FGF4

UBXN8

RUNX2

TCF7L2

CLDN1

SOX2

PTGS2

BTRC

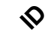

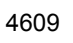

960

8839

8061

6615

319101

2697

27122

896

3889

1052

1956

1046

3671

1490

3891

7088

1044

3856

7074

1958

4233

196

8913

3866

25984

390792

1601

2114

3881

5979

80700

64220

23190

51350

137886

4035

3857

2249

7993

860

6934

9076

6657

5743

8945

Figure I FH535 suppressed the $\mathrm{Wnt} / \mathrm{\beta}$-catenin pathway in pancreatic cancer cells. Notes: (A) Time-dependent decrease by $\mathrm{FH} 535$ of nuclear and total $\beta$-catenin protein levels in PANC-I cells; FH535 did not affect nuclear or total $\beta$-catenin expression in BxPC-3 cells. (B) Dose-dependent decrease by FH535 of TCFdependent transcription. ${ }^{*} * P<0.0 \mathrm{I}$, significant differences vs the respective control groups. (C) Microarray analysis of expression regulation of genes downstream of the $\mathrm{Wnt} / \beta$-catenin pathway upon $20 \mu \mathrm{M} \mathrm{FH} 535$ treatment. Up and down arrows indicate gene expression significantly upregulated or downregulated, respectively, by twofold. Abbreviations: TCF, T-cell factor; h, hours.

Table I Microarray analysis of expression regulation of genes downstream of the $\mathrm{Wnt} / \beta$-catenin pathway upon $20 \mu \mathrm{M}$ FH535 treatment

\begin{tabular}{|c|c|c|c|}
\hline \multirow[t]{2}{*}{ Gene } & \multirow[t]{2}{*}{ ID } & \multicolumn{2}{|c|}{ Normalized intensity } \\
\hline & & Control & FH535 \\
\hline MYC & 4609 & 16.268158 & 15.204586 \\
\hline KRTI8 & 3875 & $|5.97500|$ & 16.022995 \\
\hline PTTGI & 9232 & 15.680945 & I5.73604 \\
\hline ANGPTL4 & 51129 & 15.190848 & 15.278334 \\
\hline KRT8I & 3887 & 15.0413 & 14.423697 \\
\hline$C D 44$ & 960 & 15.006962 & 16.199093 \\
\hline PFDN5 & 5204 & |4.87926| & 14.964103 \\
\hline KRTIO & 3858 & |4.79975| & |3.88979| \\
\hline PTTG2 & 10744 & | 4.772796 & $\mid 4.547727$ \\
\hline BIRC5 & 332 & | 4.757564 & 14.219355 \\
\hline PTTGIIP & 754 & 14.684395 & 14.533192 \\
\hline VEGFB & 7423 & 14.498004 & 13.671163 \\
\hline CYR6I & 3491 & |4.279853 & 14.790296 \\
\hline UBXNI & 51035 & $|4.23| 482$ & 14.049252 \\
\hline KRT7 & 3855 & |4.184294 & 13.285099 \\
\hline WISP2 & 8839 & | 3.732449 & 12.493675 \\
\hline sox9 & 6662 & | 3.574989 & $|3.4| 5|7|$ \\
\hline EN2 & 2020 & 13.393019 & 12.721889 \\
\hline JAGI & 182 & 12.427784 & 12.687155 \\
\hline FOSLI & 8061 & 12.344017 & II.102832 \\
\hline MYCBP & 26292 & $|2.28465|$ & | |.97478| \\
\hline SNAII & 6615 & 12.28132 & 10.385736 \\
\hline KRT73 & 319101 & 12.23975 & II.053284 \\
\hline GJAI & 2697 & 12.226766 & 13.521647 \\
\hline IRX3 & 79191 & 12.224495 & 12.16053 \\
\hline$T B X I$ & 6899 & 12.181493 & 12.043698 \\
\hline DKK3 & $27 \mid 22$ & 12.076692 & 10.961267 \\
\hline JUN & 3725 & 12.038464 & 12.673436 \\
\hline MSLI & 339287 & 11.920114 & |l. .438548 \\
\hline KRT80 & |4450| & |I.878|8 & 12.039767 \\
\hline CCND3 & 896 & | I.576098 & 10.075832 \\
\hline CDKN2A & 1029 & II.343829 & II.097562 \\
\hline EFNBI & 1947 & II.337793 & $|0.36835|$ \\
\hline PTTG3P & 26255 & $|1.3331|$ & 10.750982 \\
\hline KRT83 & 3889 & 11.319811 & 9.89329 \\
\hline KRTI9 & 3880 & II.289505 & 11.101922 \\
\hline CEBPD & 1052 & 11.196305 & 10.068165 \\
\hline PPARD & 5467 & II.19087 & 10.731722 \\
\hline ANTXRI & 84168 & II.149265 & $|2.12257|$ \\
\hline EGFR & 1956 & II.I22326 & 12.333595 \\
\hline$C D \times 4$ & 1046 & 10.933424 & 9.588729 \\
\hline$I S L R$ & 3671 & 10.854443 & 9.725897 \\
\hline TWIST2 & $11758 \mid$ & 10.853075 & 10.129753 \\
\hline VEGFA & 7422 & 10.833399 & $|0.08787|$ \\
\hline CTGF & 1490 & 10.809845 & II.98555 \\
\hline FZD7 & 8324 & 10.711324 & 9.901575 \\
\hline KRT85 & 3891 & 10.621079 & 9.606193 \\
\hline CCNDI & 595 & $|0.54362|$ & 10.14267 \\
\hline TLEI & 7088 & 10.343489 & 9.271956 \\
\hline$C D X I$ & 1044 & 10.329419 & 9.136879 \\
\hline KRT8 & 3856 & 10.267347 & 8.319682 \\
\hline NRPI & 8829 & 10.246916 & 9.627893 \\
\hline DKKI & 22943 & 10.211538 & $|0.97995|$ \\
\hline IRSI & 3667 & 10.175792 & 10.3609915 \\
\hline MMP2 & 4313 & 10.153262 & 9.412593 \\
\hline IKBKG & 8517 & 10.132635 & 9.295626 \\
\hline TIAMI & 7074 & 10.117085 & II.627998 \\
\hline
\end{tabular}


Table I (Continued)

\begin{tabular}{|c|c|c|c|}
\hline \multirow[t]{2}{*}{ Gene } & \multirow[t]{2}{*}{ ID } & \multicolumn{2}{|c|}{ Normalized intensity } \\
\hline & & Control & FH535 \\
\hline$\overline{E G R I}$ & 1958 & 10.007974 & 8.180263 \\
\hline MET & 4233 & 9.986441 & |2.7497| \\
\hline BGLAP & 632 & 9.971276 & 9.414629 \\
\hline VEGFC & 7424 & 9.923567 & 10.21814 \\
\hline$A H R$ & 196 & 9.886938 & II.93648| \\
\hline CACNAIG & 8913 & 9.812038 & 8.560494 \\
\hline KRTI5 & 3866 & 9.7214575 & 8.709181 \\
\hline PPAP2B & 8613 & $9.7|873|$ & $9.95658 I$ \\
\hline KRT86 & 3892 & 9.707824 & 9.012362 \\
\hline KRT23 & 25984 & 9.573925 & $7.977 \mid 22$ \\
\hline$G B \times 2$ & 2637 & 9.409858 & $9.626|5|$ \\
\hline KRT39 & 390792 & 9.284486 & 7.929165 \\
\hline WNT3A & 89780 & 9.275467 & 8.68014 \\
\hline PLAUR & 5329 & 9.265003 & 8.37788 \\
\hline ID2 & 3398 & 9.226584 & 8.999163 \\
\hline MAEA & 10296 & 9.087043 & 8.91366 \\
\hline$D A B 2$ & 1601 & 9.034534 & 10.517419 \\
\hline ETS2 & 2114 & 8.999426 & $|0.44546|$ \\
\hline KRT3I & 3881 & 8.998071 & 7.96933 \\
\hline TNFRSFIIA & 8792 & 8.943393 & 9.029845 \\
\hline RET & 5979 & 8.9224615 & 7.809108 \\
\hline UBXN6 & 80700 & 8.850218 & 7.6966906 \\
\hline STRAG & 64220 & $8.746 \mid 83$ & 7.1663184 \\
\hline KLF5 & 688 & 8.6543455 & 8.714795 \\
\hline KRT4 & 3851 & 8.640165 & 7.6698284 \\
\hline UBXN4 & 23190 & 8.607909 & 10.505396 \\
\hline LEFI & 51176 & 8.601926 & 9.380911 \\
\hline KRT76 & 51350 & 8.571253 & 7.397269 \\
\hline$U B X N 2 B$ & 137886 & 8.2286415 & 9.982969 \\
\hline UBXNII & 91544 & $8 .|7948|$ & 7.272692 \\
\hline LRPI & 4035 & 8.175423 & 6.988433 \\
\hline$U B X N 2 A$ & 165324 & 8.133479 & 7.9562063 \\
\hline KRT9 & 3857 & 8.110517 & 7.0731263 \\
\hline$E D A$ & 1896 & 8.09645 & 7.4072337 \\
\hline KRT32 & 3882 & 8.087603 & 7.7537346 \\
\hline FGF4 & 2249 & 7.9492774 & 6.5977035 \\
\hline KRT3 & 3850 & 7.8908534 & 8.469248 \\
\hline UBXN8 & 7993 & 7.86574 & 9.013798 \\
\hline SIXI & 6495 & 7.818405 & 7.9264607 \\
\hline FOXNI & 8456 & 7.7998743 & 6.8640747 \\
\hline ETV6 & 2120 & 7.7085342 & 7.0067773 \\
\hline KRTI & 3848 & 7.5221066 & 6.7497764 \\
\hline IL8 & 3576 & 7.501872 & 6.6113296 \\
\hline NTRK2 & 4915 & 7.497469 & 7.1365094 \\
\hline RUNX2 & 860 & 7.4688272 & 8.628798 \\
\hline MMPII & 4320 & 7.460847 & 7.2920337 \\
\hline $\mathrm{CDHI}$ & 999 & 7.3595057 & 7.319695 \\
\hline TCF7L2 & 6934 & $7.3556 \mid 23$ & 8.6040535 \\
\hline KRT78 & 196374 & 7.349466 & 6.8676143 \\
\hline TCF7 & 6932 & 7.270456 & 7.664296 \\
\hline SMO & 6608 & 7.222788 & 7.0400887 \\
\hline EFNB2 & 1948 & 7.1960526 & $7.2677 \mid$ \\
\hline CLDNI & 9076 & 7.1643777 & $8.94399 \mid$ \\
\hline KRT33A & 3883 & 7.121948 & 6.808277 \\
\hline VCAN & 1462 & 7.045421 & 6.763195 \\
\hline MMPQ & 4318 & 7.0101504 & 6.7540355 \\
\hline DLLI & 28514 & 6.969655 & 6.5782347 \\
\hline KRTI3 & 3860 & 6.949356 & 5.971072 \\
\hline IGF2 & 3481 & 6.933426 & 6.170534 \\
\hline
\end{tabular}

Table I (Continued)

\begin{tabular}{llll}
\hline Gene & ID & \multicolumn{2}{l}{ Normalized intensity } \\
\cline { 3 - 4 } & & Control & FH535 \\
\hline KRT26 & 353288 & 6.869997 & 6.697632 \\
TNFRSF9 & 3604 & 6.862919 & 6.6031585 \\
KRT74 & I2I39I & 6.778076 & 6.538765 \\
TWISTI & $729 I$ & 6.765423 & 6.105777 \\
NRCAM & 4897 & 6.677019 & 6.7818675 \\
FGF9 & 2254 & 6.6647215 & 5.7855196 \\
TNFRSIIB & 4982 & 6.6092443 & 6.618697 \\
CHLI & 10752 & 6.6082654 & 6.3569694 \\
KRT34 & 3885 & 6.601664 & 6.199431 \\
KRT6A & 3853 & 6.536037 & 5.9656916 \\
EDNI & 1906 & 6.476451 & 6.7537594 \\
NOS2 & 4843 & $6.42546 I$ & 6.333558 \\
GDF5 & 8200 & 6.3569694 & 6.3291264 \\
CCND2 & 894 & 6.3239446 & 5.996339 \\
DLKI & 8788 & 6.2332454 & 6.884508 \\
KRT37 & 8688 & 5.971611 & 5.881136 \\
IL6 & 3569 & 5.7313643 & 5.9466343 \\
SOX2 & 6657 & 5.6166873 & 6.7975965 \\
TGFB3 & 7043 & 5.5891886 & 6.0552535 \\
KRT35 & 3886 & 5.5883365 & 6.3580856 \\
PTGS2 & 5743 & 5.5262737 & $7.60154 I$ \\
BTRC & 8945 & 5.3152456 & 7.7473273 \\
\hline
\end{tabular}

downregulated the protein level of total $\beta$-catenin in the PANC-1 cells, which differed from the results of most previous studies. ${ }^{10}$ This cell type-dependent downregulation of $\beta$-catenin could have been due to the stabilization of axin, which suppresses $\beta$-catenin. ${ }^{11}$ Axin is characterized as a tumor-suppressor gene, and it plays a key role in inhibiting the canonical Wnt pathway by forming molecular complexes with other proteins such as GSK-3 $\beta$ and adenomatous polyposis coli (APC). ${ }^{38}$ Whether or not $\beta$-catenin expression was inhibited, the luciferase reporter assay proved that transcriptional activity of $\beta$-catenin pathway was decreased, which was consistent with previous study findings. ${ }^{10}$

Metastasis, the leading cause of cancer-related death, is a complex process comprising several steps, all of which we found were affected by FH535. First, FH535 inhibited pancreatic cancer cell migration. Microarray analyses revealed that FH535 altered the expression of several migrationrelated genes, which participate in focal adhesion, adhesion junctions, tight junctions, and/or motility regulation. Among these genes, the focal adhesion-related gene PTEN, considered "the most highly mutated tumor-suppressor gene in the post-p53 era", ${ }^{39}$ plays a role in controlling cell migration. ${ }^{40}$ The loss of PTEN protein expression or function has been reported in many human cancers, including ovarian, endometrial, and prostate carcinoma; breast cancer; and primary gastrointestinal stromal tumor. ${ }^{41,42}$ We also found that FH535 downregulated the adhesion junction-related gene TLN1, 
A

PANC-1

BXPC-3
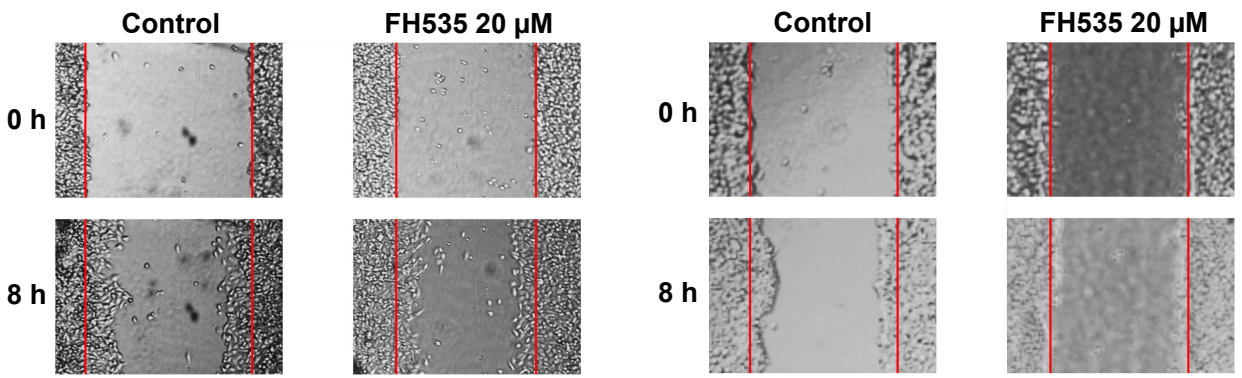

$12 \mathrm{~h}$
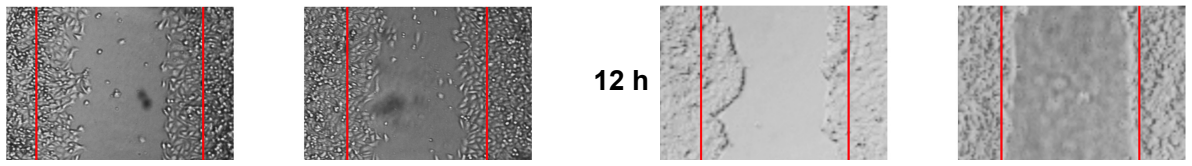

$8 \mathrm{~h}$

$12 \mathrm{~h}$
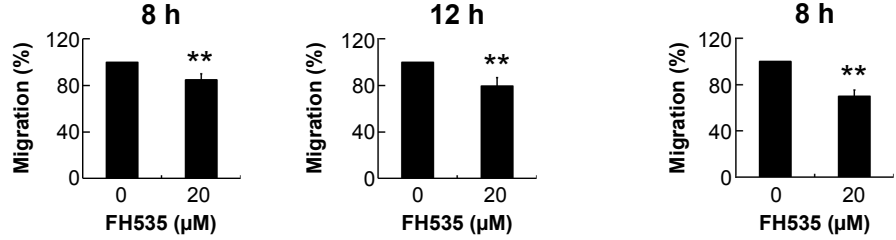

$12 \mathrm{~h}$

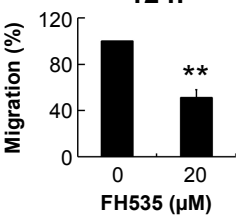

B

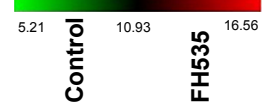

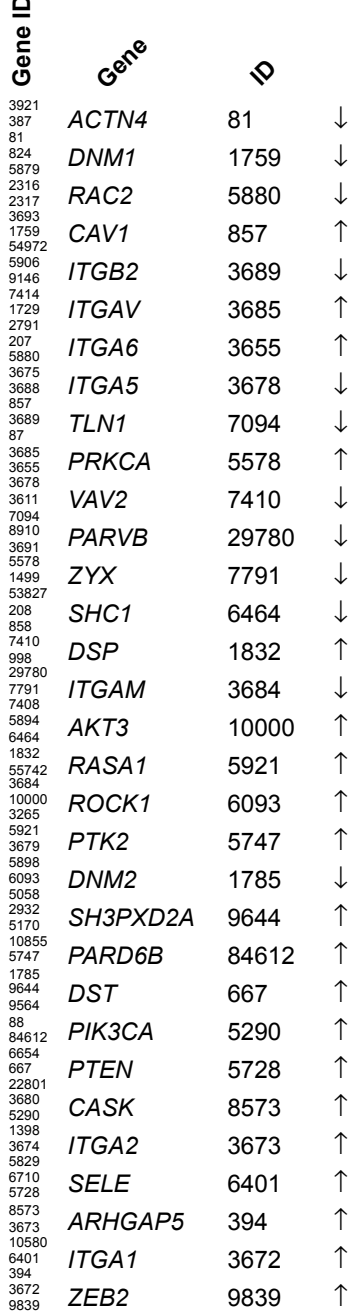

C

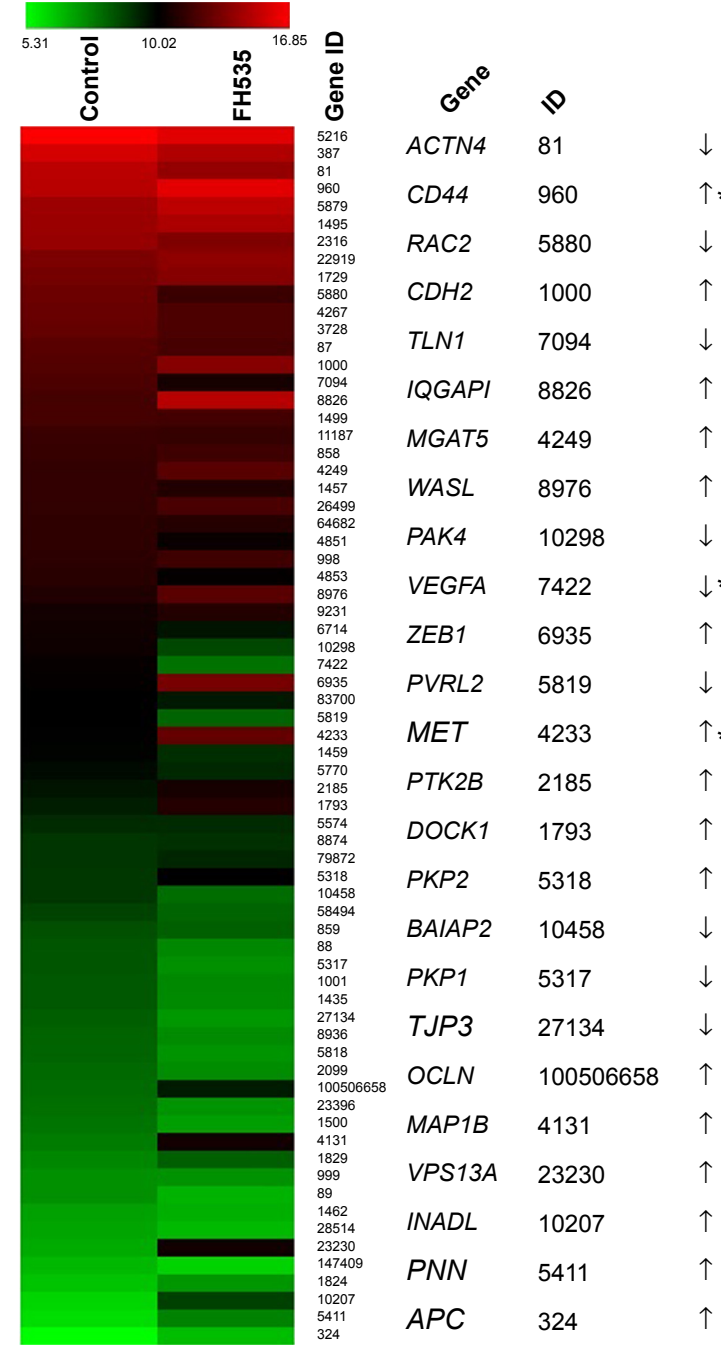

Figure 2 (Continued) 


\section{D}

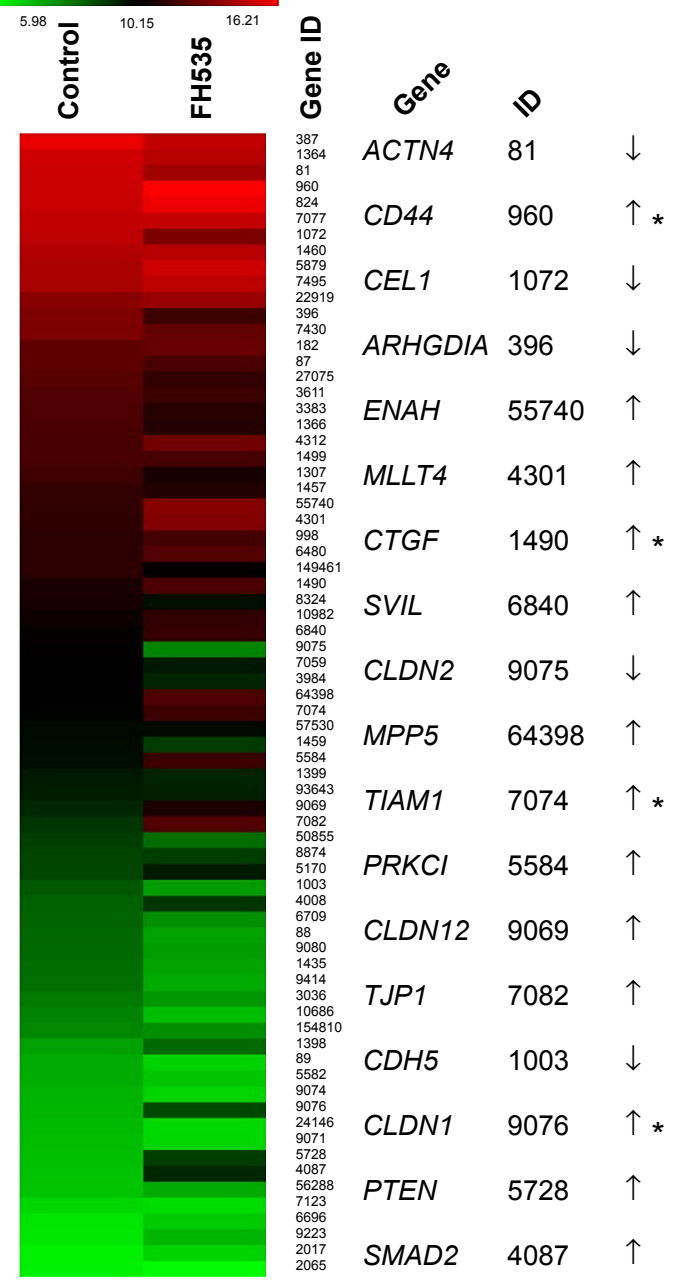

\section{E}
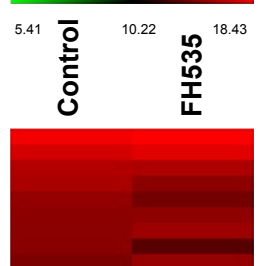

민

$\mathrm{C}^{\mathrm{O}^{\circ}}$
ACTN4
CFL1

RAP1B

RHOC

LAMC1

ACTR3

RAC2

ITGB2

PRKCA

ENAH

EGFR

WASL

CALD1

TGFB1

$R D X$

ARF6

SVIL

VEGFA

CAPN1

F11R

RND3

WASF2

FAT1

RHOB

RAPGEF1

RASA1

PTK2B

ROCK1

MYH10

MMP15

DOCK1

PAK2

CDC27

BAIAP2

PTK2

PKP4

PVRL3

OCLN

DOCK4

MAP1B

MTSS1

EXOC2

CASK

\begin{tabular}{ll} 
叉 & \\
81 & $\downarrow$ \\
1072 & $\downarrow$ \\
5908 & $\downarrow$ \\
389 & $\uparrow$ \\
3915 & $\downarrow$ \\
10096 & $\uparrow$ \\
5880 & $\uparrow$ \\
3689 & $\downarrow$ \\
5578 & $\downarrow$ \\
55740 & $\uparrow$ \\
1956 & $\uparrow$ \\
8976 & $\uparrow$ \\
800 & $\uparrow$ \\
7040 & $\uparrow$ \\
5962 & $\downarrow$ \\
382 & $\uparrow$ \\
6840 & $\uparrow$ \\
7422 & $\uparrow$ \\
823 & $\downarrow$ \\
50848 & $\downarrow$ \\
390 & $\downarrow$ \\
10163 & $\downarrow$ \\
2195 & $\uparrow$ \\
388 & $\uparrow$ \\
2889 & $\downarrow$ \\
5921 & $\uparrow$ \\
2185 & $\uparrow$ \\
6093 & $\uparrow$ \\
4628 & $\uparrow$ \\
4324 & $\uparrow$ \\
1793 & $\uparrow$ \\
5062 & $\uparrow$ \\
996 & $\uparrow$ \\
5747 \\
8502 & $\uparrow$ \\
25945 & $\uparrow$ \\
100506658 & $\uparrow$ \\
9732 & $\uparrow$ \\
9131 & $\uparrow$ \\
55770 & $\uparrow$ \\
8573 & $\uparrow$ \\
& $\uparrow$ \\
\hline
\end{tabular}

Figure 2 FH535 inhibited pancreatic cancer cell migration.

Notes: (A) Time-dependent inhibition by FH535 of PANC-I and BxPC-3 cell migration. ${ }^{* * P}<0.0$ I, significant differences vs the respective control groups. Microarray analysis of (B) focal adhesion-related, (C) adhesion junction-related, (D) tight junction-related, and (E) cell motility-related gene expression regulation upon FH535 treatment. Up and down arrows indicate gene expression significantly upregulated or downregulated, respectively, by twofold. Asterisks indicate genes downstream of the Wnt/ $\beta$-catenin pathway.

Abbreviation: $\mathrm{h}$, hours.

which encodes a cytoskeletal protein that is concentrated in areas of cell-substratum and cell-cell contact. The encoded protein plays a significant role in actin filament assembly and in the spread and migration of various cell types. ${ }^{43,44}$ TLN1 is codistributed with integrins in the cell surface membrane, aiding the attachment of adherent cells to extracellular matrices and lymphocytes to other cells. In our study, tight junction protein 1 (TJP1), which plays a critical role in cell-cell interaction, proliferation, and differentiation, was upregulated. TJP1 is an important marker of tight junction integrity, which is disrupted in many highly invasive cancers; upregulated TJP1 correlates with favorable survival 
Table 2 Microarray analysis of focal adhesion-related gene expression regulation upon $\mathrm{FH} 535$ treatment

\begin{tabular}{|c|c|c|c|c|c|c|c|}
\hline \multicolumn{4}{|c|}{ expression regulation upon $\mathrm{FH} 535$ treatment } & \multirow[t]{2}{*}{ Gene } & \multirow[t]{2}{*}{ ID } & \multicolumn{2}{|c|}{ Normalized intensity } \\
\hline \multirow[t]{2}{*}{ Gene } & \multirow[t]{2}{*}{ ID } & \multicolumn{2}{|c|}{ Normalized intensity } & & & Control & FH535 \\
\hline & & Control & FH535 & ITGAII & 22801 & 7.7725782 & 7.250522 \\
\hline RPSA & 3921 & 16.551584 & 16.069508 & ITGA9 & 3680 & 7.725706 & 7.2797456 \\
\hline RHOA & 387 & 15.761177 & $|4.78665|$ & PIKЗСА & 5290 & 7.546133 & 9.475706 \\
\hline ACTN4 & 81 & 15.032014 & $|4.0| 403$ & CRK & 1398 & 7.5114365 & 8.404298 \\
\hline CAPN2 & 824 & 14.947017 & $15.84|3| 4$ & ITGA2B & 3674 & 7.474538 & 6.8249826 \\
\hline RACI & 5879 & 14.251518 & 15.113209 & $P X N$ & 5829 & 7.426979 & 6.493304 \\
\hline FLNA & 2316 & 14.083586 & 13.488903 & SPTB & 6710 & 7.143782 & 6.598218 \\
\hline FLNB & 2317 & | 3.958575 & 13.296808 & PTEN & 5728 & 7.0376005 & 9.120998 \\
\hline ITGB5 & 3693 & | 3.888797 & | 3.484464 & CASK & 8573 & 6.554297 & 9.147331 \\
\hline DNMI & 1759 & $|3.64009|$ & $|2.5| 882 \mid$ & ITGA2 & 3673 & $6.53814 \mathrm{I}$ & 10.171594 \\
\hline TMEM/32A & 54972 & 13.622586 & 13.150153 & SORBSI & 10580 & 6.5160394 & 7.080136 \\
\hline RAPIA & 5906 & 13.5597315 & 14.039375 & SELE & 6401 & 5.8820415 & 7.629673 \\
\hline HGS & 9146 & | 3.533683 & | 3.846248 & ARHGAP5 & 394 & 5.628543 & 7.9602804 \\
\hline$V C L$ & 7414 & | 3.376745 & $13.68 \mid 126$ & ITGAI & 3672 & 5.3547735 & 7.6031985 \\
\hline DIAPHI & 1729 & 13.16062 & | 3.659487 & ZEB2 & 9839 & 5.2203803 & 6.942315 \\
\hline GNGII & 2791 & | 3.022779 & | 3.403848 & & & & \\
\hline AKTI & 207 & 12.957863 & 12.259176 & & & & \\
\hline RAC2 & 5880 & $12.9550 \mid 5$ & 11.604415 & & & & \\
\hline ITGA3 & 3675 & 12.797894 & 12.391577 & \multirow{2}{*}{\multicolumn{4}{|c|}{$\begin{array}{l}\text { Table } 3 \text { Microarray analysis of adhesion junction-related gene } \\
\text { expression regulation upon FH535 treatment }\end{array}$}} \\
\hline ITGBI & 3688 & 12.738785 & 13.636554 & & & & \\
\hline $\begin{array}{l}\text { CAVI } \\
\text { CTCR2 }\end{array}$ & $\begin{array}{l}857 \\
3689\end{array}$ & $\begin{array}{l}12.61244 \\
12546266\end{array}$ & $\begin{array}{l}13.617725 \\
1473748\end{array}$ & \multirow[t]{2}{*}{ Gene } & \multirow[t]{2}{*}{ ID } & \multicolumn{2}{|c|}{ Normalized intensity } \\
\hline $\begin{array}{l}\text { ITGB2 } \\
\text { ACTNI }\end{array}$ & $\begin{array}{l}3689 \\
87\end{array}$ & $\begin{array}{l}12.546266 \\
12.409878\end{array}$ & $\begin{array}{l}\text { II.473748 } \\
\text { II.967234 }\end{array}$ & & & Control & FH535 \\
\hline ITGAV & 3685 & 12.278682 & $\mid 4.3424$ & PFNI & 5216 & 16.843973 & 16.144138 \\
\hline ITGA6 & 3655 & I2.273888 & $|4.3924| 8$ & RHOA & 387 & $15.76 \mid 177$ & $|4.78665|$ \\
\hline ITGA5 & 3678 & 12.169847 & 10.866323 & ACTN4 & 81 & $15.0320 \mid 4$ & $|4.0| 403$ \\
\hline ILK & 3611 & 12.11682 & II.583433 & CD44 & 960 & 15.006962 & 16.199093 \\
\hline TLNI & 7094 & $|2.09664|$ & 10.645829 & RACI & 5879 & $|4.25| 5 \mid 8$ & $15.1 \mid 3209$ \\
\hline SGCE & 8910 & 12.047686 & $|2.28232|$ & CTNNAI & 1495 & 14.209974 & 14.654735 \\
\hline ITGB4 & 3691 & 11.982763 & II.56935 & FLNA & 2316 & 14.083586 & 13.488903 \\
\hline PRKCA & 5578 & 11.918201 & 13.803304 & MAPREI & 22919 & $|3.4| 3|4|$ & | 3.8757925 \\
\hline CTNNBI & 1499 & 11.900537 & 11.841962 & DIAPHI & 1729 & 13.16062 & 13.659487 \\
\hline FXYD5 & 53827 & II.859393 & 10.980669 & RAC2 & 5880 & 12.955015 & 11.604415 \\
\hline$A K T 2$ & 208 & 11.791592 & 10.995004 & CD99 & 4267 & 12.8705635 & 12.11682 \\
\hline CAV2 & 858 & II.534644 & 11.731664 & JUP & 3728 & 12.776809 & 12.098349 \\
\hline VAV2 & 7410 & II.322939 & 10.17948 & ACTNI & 87 & 12.409878 & II.967234 \\
\hline$C D C 42$ & 998 & II.250544 & II.791042 & $\mathrm{CDH} 2$ & 1000 & 12.27524 & 13.657263 \\
\hline PARVB & 29780 & II.224628 & 9.830263 & TLNI & 7094 & $|2.09664|$ & 10.645829 \\
\hline$Z Y X$ & 7791 & 10.997072 & 9.663998 & IQGAPI & 8826 & 11.903805 & I5.008826 \\
\hline VASP & 7408 & 10.877319 & 10.418066 & CTNNBI & 1499 & 11.900537 & 11.841962 \\
\hline RAFI & 5894 & 10.594473 & 10.865986 & PKP3 & 11187 & II.572304 & II.483009 \\
\hline $\mathrm{SHCl}$ & 6464 & 10.287678 & 8.595637 & CAV2 & 858 & II.534644 & 11.731664 \\
\hline$D S P$ & 1832 & 10.226259 & II.500326 & MGAT5 & 4249 & II.399225 & I2.44798 \\
\hline PARVA & 55742 & $10.08046 \mid 5$ & 10.301352 & CSNK2AI & 1457 & II.389523 & 10.994029 \\
\hline ITGAM & 3684 & 10.003317 & 8.889115 & PLEK2 & 26499 & II.380254 & 12.049034 \\
\hline AKT3 & 10000 & 9.999831 & $12.07 \mid 189$ & ANAPCI & 64682 & 11.330902 & II.025982 \\
\hline HRAS & 3265 & 9.972342 & 9.272375 & NOTCHI & $485 I$ & 11.311136 & $|0.345| 43$ \\
\hline PDPKI & 5170 & 9.005413 & 9.698432 & $C D C 42$ & 998 & II.250544 & II.791042 \\
\hline HPSE & 10855 & 8.978405 & 8.067395 & NOTCH2 & 4853 & II.I25797 & 10.202223 \\
\hline PTK2 & 5747 & 8.939062 & 10.820772 & WASL & 8976 & 10.930079 & I2.483249 \\
\hline DNM2 & 1785 & 8.613899 & 7.43392 & DLG5 & 9231 & 10.567565 & 11.019769 \\
\hline$S H 3 P X D 2 A$ & 9644 & 8.566784 & 9.949804 & $S R C$ & 6714 & $10.48 \mid 47$ & 9.648777 \\
\hline BCARI & 9564 & 8.529809 & 7.7692404 & PAK4 & 10298 & 10.446864 & 8.676079 \\
\hline ACTN2 & 88 & 8.437073 & 7.474538 & VEGFA & 7422 & 10.250756 & 7.901348 \\
\hline PARD6B & 84612 & 8.172608 & 9.636746 & ZEBI & 6935 & 10.177025 & | 3.283847 \\
\hline SOSI & 6654 & 7.9648976 & 7.5355105 & JAM3 & 83700 & 10.084784 & 9.556893 \\
\hline DST & 667 & 7.7908773 & II.245214 & PVRL2 & 5819 & 10.018614 & 8.147698 \\
\hline & & & (Continued) & & & & (Continued) \\
\hline
\end{tabular}

Table 2 (Continued)

Table 3 Microarray analysis of adhesion junction-related gene xpression regulation upon $\mathrm{FH} 535$ treatment 
Table 3 (Continued)

\begin{tabular}{|c|c|c|c|}
\hline \multirow[t]{2}{*}{ Gene } & \multirow[t]{2}{*}{ ID } & \multicolumn{2}{|c|}{ Normalized intensity } \\
\hline & & Control & FH535 \\
\hline$\overline{M E T}$ & 4233 & $9.98644 I$ & $|2.7497|$ \\
\hline CSNK2A2 & 1459 & 9.960693 & 9.169151 \\
\hline PTPNI & 5770 & 9.82763 & 9.271097 \\
\hline PTK2B & 2185 & 9.636893 & 10.68203 \\
\hline DOCKI & 1793 & 9.48097 & 11.021774 \\
\hline MAPKI & 5594 & 9.240688 & 9.218932 \\
\hline ARHGEF7 & 8874 & 9.037083 & 9.147919 \\
\hline CBLLI & 79872 & 9.0251875 & 9.311203 \\
\hline PKP2 & 5318 & 9.022291 & $|0.02578|$ \\
\hline BAIAP2 & 10458 & 9.018734 & 8.004229 \\
\hline JAM2 & 58494 & 8.78947I & 8.162707 \\
\hline CAV3 & 859 & 8.535716 & 8.229119 \\
\hline ACTN2 & 88 & 8.437073 & 7.474538 \\
\hline PKPI & 5317 & 8.423988 & 7.3570046 \\
\hline $\mathrm{CDH} 3$ & 1001 & 8.389479 & 7.50349 \\
\hline CSFI & 1435 & 8.360545 & 7.4550886 \\
\hline TJP3 & 27134 & 8.295799 & 7.207067 \\
\hline WASFI & 8936 & 8.178464 & 7.4462004 \\
\hline PVRLI & 5818 & 8.152037 & 7.283169 \\
\hline ESRI & 2099 & 8.048168 & 7.405365 \\
\hline OCLN & 100506658 & 8.036663 & 9.494983 \\
\hline PIP5KIC & 23396 & 7.9606485 & 7.254657 \\
\hline CTNNDI & 1500 & 7.8659673 & 7.09317 \\
\hline MAPIB & $4|3|$ & 7.7052383 & 10.589897 \\
\hline DSG2 & 1829 & 7.513804 & 8.2605915 \\
\hline $\mathrm{CDHI}$ & 999 & 7.3595057 & 7.319695 \\
\hline ACTN3 & 89 & 7.355976 & 6.6988516 \\
\hline VCAN & 1462 & 7.045421 & 6.763195 \\
\hline DLLI & 28514 & 6.969655 & 6.5782347 \\
\hline VPSI $3 A$ & 23230 & 6.859817 & 10.562696 \\
\hline DSG4 & |47409 & 6.608555 & 6.1116643 \\
\hline DSC2 & 1824 & 6.3962626 & 7.2484 I \\
\hline INADL & 10207 & 6.08029 & 8.808925 \\
\hline PNN & 5411 & 5.9342465 & 7.5790677 \\
\hline$A P C$ & 324 & 5.3153567 & 6.5241365 \\
\hline ITGA2 & 3673 & $6.538|4|$ & 10.171594 \\
\hline SORBSI & 10580 & 6.5160394 & 7.080136 \\
\hline SELE & 6401 & 5.8820415 & 7.629673 \\
\hline ARHGAP5 & 394 & 5.628543 & 7.9602804 \\
\hline ITGAI & 3672 & 5.3547735 & 7.6031985 \\
\hline ZEB2 & 9839 & 5.2203803 & 6.942315 \\
\hline
\end{tabular}

Table 4 Microarray analysis of tight junction-related gene expression regulation upon $\mathrm{FH} 535$ treatment

\begin{tabular}{llll}
\hline Gene & ID & \multicolumn{2}{l}{ Normalized intensity } \\
\cline { 3 - 4 } & & Control & FH535 \\
\hline RHOA & 387 & 15.761177 & $14.78665 \mid$ \\
CLDN4 & 1364 & 15.11957 & 14.539507 \\
ACTN4 & $8 I$ & 15.032014 & 14.01403 \\
CD44 & 960 & 15.006962 & 16.199093 \\
CAPN2 & 824 & 14.947017 & 15.841314 \\
TIMP2 & 7077 & 14.796619 & 14.858342 \\
CFLI & 1072 & 14.710272 & 13.114106 \\
CSNK2B & 1460 & 14.4039135 & 14.575101 \\
RACI & 5879 & 14.251518 & 15.113209 \\
CTNNAI & 1495 & 14.209974 & 14.654735 \\
MAPREI & 22919 & 13.413141 & 13.8757925 \\
ARHGDIA & 396 & 13.207352 & 11.634186 \\
\hline & & & (Continued)
\end{tabular}

Table 4 (Continued)

\begin{tabular}{|c|c|c|c|}
\hline \multirow[t]{2}{*}{ Gene } & \multirow[t]{2}{*}{ ID } & \multicolumn{2}{|c|}{ Normalized intensity } \\
\hline & & Control & FH535 \\
\hline$\overline{E Z R}$ & 7430 & |3.144885 & $|2.56693|$ \\
\hline JAGI & 182 & 12.427784 & 12.687155 \\
\hline ACTNI & 87 & 12.409878 & II.967234 \\
\hline TSPAN $/ 3$ & 27075 & 12.246902 & | I.37938| \\
\hline ILK & 3611 & 12.11682 & II.583433 \\
\hline ICAMI & 3383 & 12.0056095 & 11.118488 \\
\hline CLDN7 & 1366 & 11.972866 & II.032687 \\
\hline$M M P I$ & 4312 & 11.905035 & 12.887484 \\
\hline CTNNBI & 1499 & 11.900537 & 11.841962 \\
\hline COLI6AI & 1307 & || $.64775 \mid$ & 10.777789 \\
\hline CSNK2AI & 1457 & II.389523 & 10.994029 \\
\hline ENAH & 55740 & | I.35448| & 13.398125 \\
\hline MLLT4 & 4301 & II.299263 & | 3.275789 \\
\hline $\mathrm{CDC} 42$ & 998 & II.250544 & 11.791042 \\
\hline$I G F I R$ & 3480 & 11.2369585 & 12.140446 \\
\hline CLDN/9 & $|4946|$ & 11.222952 & 10.278006 \\
\hline CTGF & 1490 & 10.809845 & II.98555 \\
\hline FZD7 & 8324 & 10.711324 & 9.901575 \\
\hline MAPRE2 & 10982 & 10.535324 & II.375724 \\
\hline SVIL & 6840 & 10.304885 & 11.463148 \\
\hline CLDN2 & 9075 & 10.221999 & 7.959734 \\
\hline THBS3 & 7059 & 10.1687765 & 9.736564 \\
\hline LIMKI & 3984 & 10.151468 & 9.52237 \\
\hline MPP5 & 64398 & 10.149654 & 12.064446 \\
\hline TIAMI & 7074 & 10.117085 & II.627998 \\
\hline CGN & 57530 & 10.004088 & 9.987757 \\
\hline CSNK2A2 & 1459 & 9.960693 & 9.169151 \\
\hline PRKCI & 5584 & 9.934886 & II.633633 \\
\hline$C R K L$ & 1399 & 9.737389 & 9.574368 \\
\hline TJAPI & 93643 & 9.66933 & 9.609078 \\
\hline CLDN 12 & 9069 & 9.506469 & 10.83943 \\
\hline TJPI & 7082 & 9.28694 & 12.134832 \\
\hline PARD6A & 50855 & 9.12321 & 8.362814 \\
\hline ARHGEF7 & 8874 & 9.037083 & 9.147919 \\
\hline PDPKI & 5170 & 9.005413 & 9.698432 \\
\hline $\mathrm{CDH} 5$ & 1003 & 8.708324 & 7.5856657 \\
\hline LMO7 & 4008 & 8.558113 & 9.277104 \\
\hline SPTANI & 6709 & 8.494044 & 7.7864056 \\
\hline ACTN2 & 88 & 8.437073 & 7.474538 \\
\hline CLDN9 & 9080 & 8.4181795 & 7.5940213 \\
\hline CSFI & 1435 & 8.360545 & 7.4550886 \\
\hline$T J P 2$ & 9414 & 8.343918 & 7.3491254 \\
\hline HASI & 3036 & 8.124433 & 7.6573296 \\
\hline CLDN/6 & 10686 & 7.9999046 & 7.022292 \\
\hline AMOTLI & 154810 & 7.8963585 & 7.8100796 \\
\hline CRK & 1398 & 7.5114365 & 8.404298 \\
\hline ACTN3 & 89 & 7.355976 & 6.6988516 \\
\hline PRKCG & 5582 & 7.321149 & 6.9112835 \\
\hline CLDN6 & 9074 & 7.220466 & 6.6578355 \\
\hline CLDNI & 9076 & 7.1643777 & 8.943991 \\
\hline CLDN/5 & 24146 & 7.0927997 & 6.5795236 \\
\hline CLDNIO & 9071 & 7.0557775 & 6.613464 \\
\hline PTEN & 5728 & 7.0376005 & 9.120998 \\
\hline SMAD2 & 4087 & 6.9688606 & 9.496367 \\
\hline PARD3 & 56288 & 6.94016 & 7.33421 \\
\hline CLEC3B & 7123 & 6.6491346 & 6.5587797 \\
\hline SPPI & 6696 & 6.37645 & 6.842924 \\
\hline MAGII & 9223 & 6.3656254 & 7.168139 \\
\hline CTTN & 2017 & 6.2022476 & 6.6959023 \\
\hline ERBB3 & 2065 & 6.178696 & 5.9926624 \\
\hline
\end{tabular}


Table 5 Microarray analysis of cell motility-related gene expression regulation upon $\mathrm{FH} 535$ treatment

\begin{tabular}{|c|c|c|c|}
\hline \multirow[t]{2}{*}{ Gene } & \multirow[t]{2}{*}{ ID } & \multicolumn{2}{|c|}{ Normalized intensity } \\
\hline & & Control & FH535 \\
\hline VIM & 7431 & 18.111416 & 18.417988 \\
\hline PERP & 64065 & 17.034954 & 17.530819 \\
\hline MYH9 & 4627 & 16.01196 & 15.906586 \\
\hline RHOA & 387 & $15.76 \mid 177$ & $|4.78665|$ \\
\hline ACTN4 & 81 & 15.032014 & $14.0 \mid 403$ \\
\hline TIMP2 & 7077 & 14.796619 & 14.858342 \\
\hline MSN & 4478 & $|4.75| 84 \mid$ & 15.357616 \\
\hline CFLI & 1072 & 14.710272 & 13.114106 \\
\hline RACI & 5879 & 14.251518 & 15.113209 \\
\hline RAPIB & 5908 & $|4.02366|$ & 15.037672 \\
\hline TIMPI & 7076 & 13.919523 & 13.2338505 \\
\hline CDK4 & 1019 & 13.87332 & 13.635977 \\
\hline $\mathrm{RHOC}$ & 389 & 13.521647 & 12.094296 \\
\hline LAMCI & 3915 & $|3.49242|$ & 14.51922 \\
\hline$V C L$ & 7414 & | 3.376745 & $13.681 \mid 26$ \\
\hline ACTR3 & 10096 & 13.228158 & $14.456 \mid 6$ \\
\hline DIAPHI & 1729 & 13.16062 & | 3.659487 \\
\hline$E Z R$ & 7430 & 13.144885 & $|2.56693|$ \\
\hline VAPA & 9218 & 13.089962 & | 3.857084 \\
\hline AKTI & 207 & 12.957863 & 12.259176 \\
\hline RAC2 & 5880 & 12.955015 & $11.6044 \mid 5$ \\
\hline ACTR2 & 10097 & 12.94824 & 13.651513 \\
\hline ITGBI & 3688 & 12.738785 & 13.636554 \\
\hline PRKCZ & 5590 & 12.597843 & 11.836956 \\
\hline ITGB2 & 3689 & 12.546266 & II.473748 \\
\hline ACTNI & 87 & 12.409878 & II.967234 \\
\hline ILK & 3611 & 12.11682 & II.583433 \\
\hline SGCE & 8910 & 12.047686 & $|2.28232|$ \\
\hline ICAMI & 3383 & 12.0056095 & II.II8488 \\
\hline PPL & 5493 & II.998627 & 11.51075 \\
\hline PRKCA & 5578 & 11.918201 & 13.803304 \\
\hline PPPDE2 & $2735 I$ & II.624274 & | I.7742II \\
\hline ENAH & 55740 & || $.35448 \mid$ & $13.398 \mid 25$ \\
\hline$C D C 42$ & 998 & II.250544 & II.791042 \\
\hline EGFR & 1956 & II.122326 & 12.333595 \\
\hline WASL & 8976 & 10.930079 & 12.483249 \\
\hline CALDI & 800 & 10.921519 & $|2.29469|$ \\
\hline STEAPI & 26872 & $|0.89549|$ & II.820029 \\
\hline TGFBI & 7040 & 10.7152 & 9.03388 \\
\hline CAMK2NI & 55450 & 10.587699 & 10.065469 \\
\hline$R D X$ & 5962 & $|0.52225|$ & 12.191257 \\
\hline MCAM & 4162 & 10.452353 & 9.462444 \\
\hline ARF6 & 382 & $|0.4| 57||$ & II.52632 \\
\hline SVIL & 6840 & 10.304885 & 11.463148 \\
\hline RGS2 & 5997 & 10.257294 & 9.80196 \\
\hline VEGFA & 7422 & 10.250756 & 7.901348 \\
\hline CAPNI & 823 & 10.239203 & 8.216266 \\
\hline FIIR & 50848 & 10.234683 & 9.044022 \\
\hline RND3 & 390 & 10.199277 & 12.088578 \\
\hline MMP2 & 4313 & 10.153262 & 9.412593 \\
\hline WASF2 & 10163 & 10.085579 & 8.826545 \\
\hline FATI & 2195 & 9.970972 & $|2.04284|$ \\
\hline RHOB & 388 & 9.965946 & 8.545685 \\
\hline RAPGEFI & 2889 & 9.903289 & 8.354535 \\
\hline RASAI & 5921 & 9.702747 & II. $50200 \mid$ \\
\hline PTK2B & 2185 & 9.636893 & 10.68203 \\
\hline ROCKI & 6093 & 9.603488 & II.484902 \\
\hline
\end{tabular}

(Continued)
Table 5 (Continued)

\begin{tabular}{|c|c|c|c|}
\hline \multirow[t]{2}{*}{$\overline{\text { Gene }}$} & \multirow[t]{2}{*}{ ID } & \multicolumn{2}{|c|}{ Normalized intensity } \\
\hline & & Control & FH535 \\
\hline$\overline{M Y H I O}$ & 4628 & 9.5496025 & 11.096066 \\
\hline MMPI5 & 4324 & 9.533566 & 8.395943 \\
\hline DOCKI & 1793 & 9.48097 & 11.021774 \\
\hline PAK2 & 5062 & 9.287464 & 10.61245 \\
\hline PLAUR & 5329 & 9.265003 & 8.37788 \\
\hline$C D C 27$ & 996 & 9.129515 & II.077047 \\
\hline$M S T I R$ & 4486 & 9.085802 & 9.12538 \\
\hline BAIAP2 & 10458 & 9.018734 & 8.004229 \\
\hline PTK2 & 5747 & 8.939062 & 10.820772 \\
\hline STAT3 & 6774 & 8.847785 & 7.9192953 \\
\hline ARHGEF2 & 9181 & 8.798216 & 8.383045 \\
\hline PKP4 & 8502 & 8.663319 & 9.734335 \\
\hline MARK2 & 2011 & 8.612933 & 8.00314 \\
\hline PVRL3 & 25945 & 8.582907 & 9.851074 \\
\hline BCARI & 9564 & 8.529809 & 7.7692404 \\
\hline$A R V C F$ & 421 & 8.524339 & 8.364944 \\
\hline SPTANI & 6709 & 8.494044 & 7.7864056 \\
\hline TJP2 & 9414 & 8.343918 & $7.349 \mid 254$ \\
\hline HCLSI & 3059 & 8.263556 & 7.7210197 \\
\hline WASFI & 8936 & 8.178464 & 7.4462004 \\
\hline HASI & 3036 & 8.124433 & 7.6573296 \\
\hline ADAMTSI 3 & 11093 & 8.074093 & 8.375932 \\
\hline ESRI & 2099 & 8.048168 & 7.405365 \\
\hline OCLN & 100506658 & 8.036663 & 9.494983 \\
\hline WAS & 7454 & 7.9598556 & 7.412658 \\
\hline CTNNDI & 1500 & 7.8659673 & 7.09317 \\
\hline DOCK4 & 9732 & 7.829811 & 10.702755 \\
\hline CDSN & $104 \mid$ & 7.738298 & 7.3062844 \\
\hline MAPIB & $4|3|$ & 7.7052383 & 10.589897 \\
\hline MMPII & 4320 & 7.460847 & 7.2920337 \\
\hline PXN & 5829 & 7.426979 & 6.493304 \\
\hline ACTN3 & 89 & 7.355976 & 6.6988516 \\
\hline MTSSI & 9788 & 7.3144355 & 8.826939 \\
\hline VCAN & 1462 & 7.045421 & 6.763195 \\
\hline MMPQ & 4318 & 7.0101504 & 6.7540355 \\
\hline VTN & 7448 & 6.8925853 & 6.3992944 \\
\hline EXOC2 & 55770 & 6.8692775 & 8.335709 \\
\hline$E C M I$ & 1893 & 6.8224096 & 7.0186477 \\
\hline TWISTI & 7291 & 6.765423 & 6.105777 \\
\hline ADAMTSI & 9510 & 6.670437 & 7.5566187 \\
\hline CASK & 8573 & 6.554297 & $9 . \mid 47331$ \\
\hline PLCGI & 5335 & 6.326862 & 6.175169 \\
\hline CTTN & 2017 & 6.2022476 & 6.6959023 \\
\hline FARP2 & 9855 & 5.4352922 & 5.775334 \\
\hline CTNND2 & $|50|$ & 5.4170265 & 5.9111185 \\
\hline
\end{tabular}

in breast cancer and gastrointestinal stromal tumor. ${ }^{45,46}$ The motility-related gene VEGFA significantly increases the motility of pancreatic cancer cells. The vascular endothelial growth factor/vascular endothelial growth factor receptor (VEGF/VEGFR) inhibitors bevacizumab and sunitinib significantly decrease pancreatic cancer cell motility. ${ }^{47}$ In our study, FH535 not only suppressed VEGFA expression 
A

PANC-1

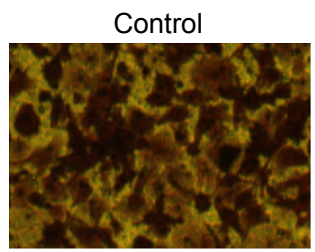

FH535 $20 \mu \mathrm{M}$

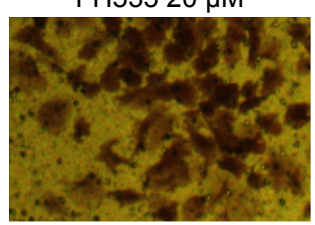

FH535 $40 \mu \mathrm{M}$

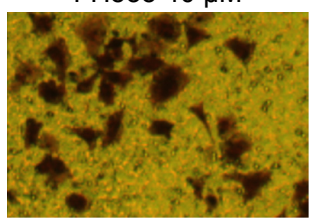

BxPC-3

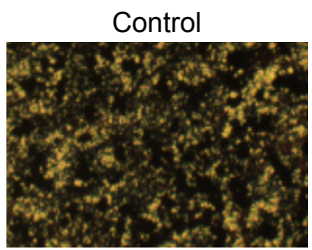

FH535 $20 \mu \mathrm{M}$

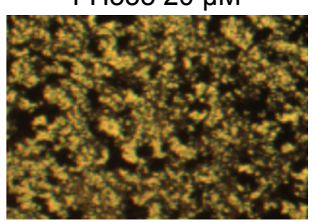

FH535 $40 \mu \mathrm{M}$

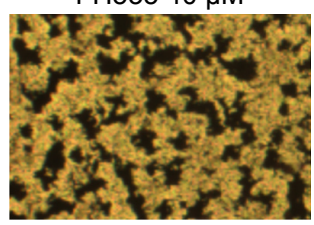

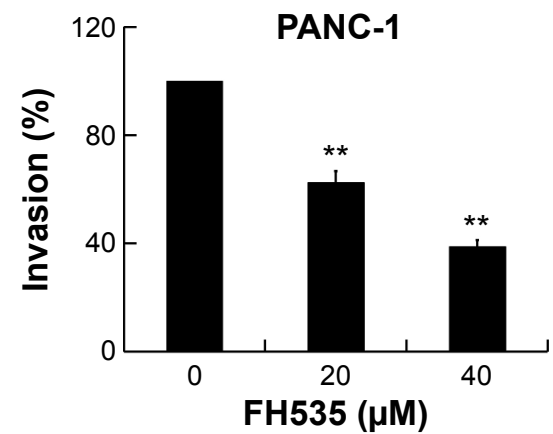

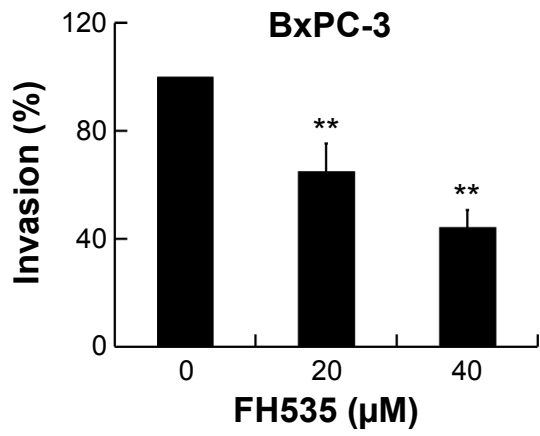

B

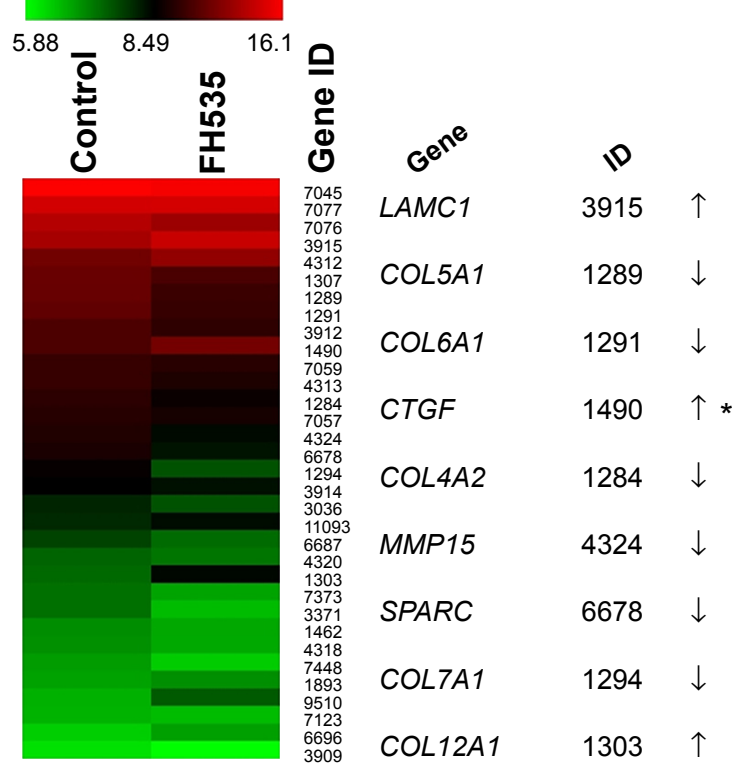

C
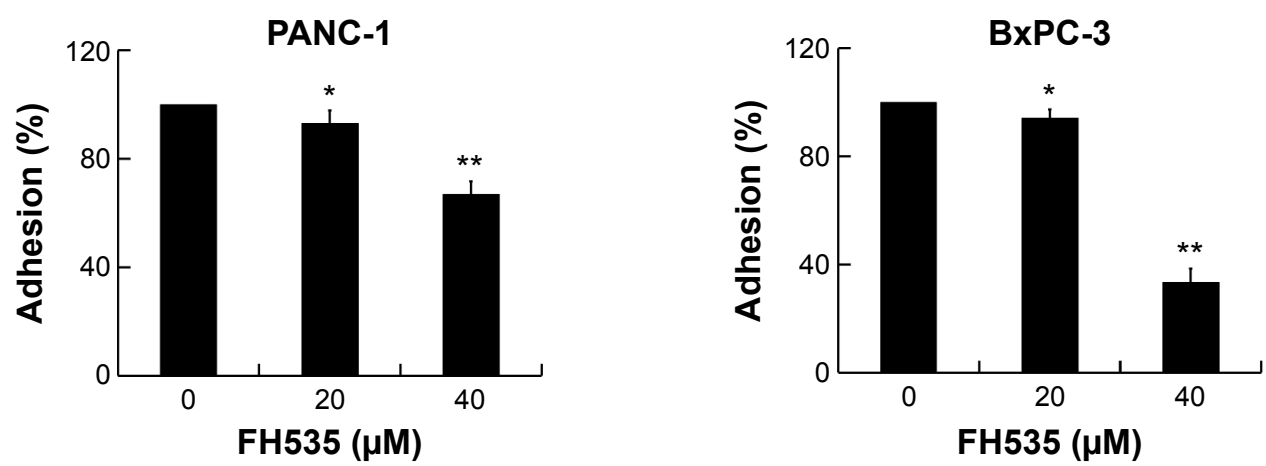

Figure 3 (Continued) 


\section{D}
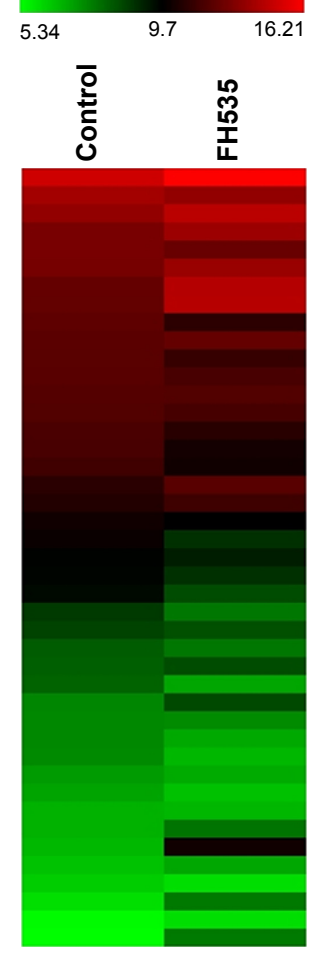

$G^{e^{n e}}$

CD44

$\angle A M C 1$

ITGAV

ITGA6

ITGA5

COL5A1

COL6A1

CTGF

ITGAM

MMP15

COL7A1

SPG7

COL12A1

ITGB2

ITGA2

SELE

ITGA1

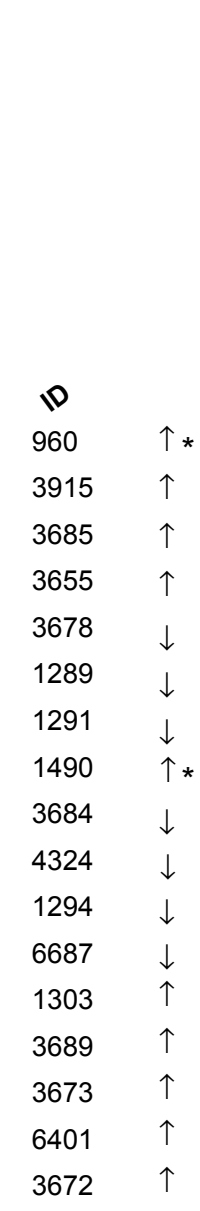

\section{E}

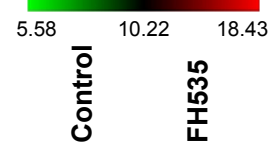

응

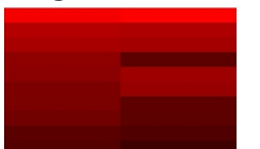

$G^{e^{n}} \quad D$

IGFBP4 3487

CAV1 857

SNAl1 6615

ITGA5 3678

$\begin{array}{ll}T L N 1 & 7094\end{array}$

COL5A1 1289

NOTCH 34854

COL6A1 1291

EGFR 1956

WASL $\quad 8976$

CALD1 800

TGFB1 $\quad 7040$

GRB2 2885

RDX $\quad 5962$

SVIL

PLEC

SHC1

SSX2IP

COL $4 A 2$

SPARC

VEZT

ZAK

COL7A1

SPG7

COL12A1

PTEN

MPP6

$B M P 2$

SOS2

6840

5339

6464

6687

1303

5728

51678

650

6655

$117178 \uparrow$

$1284 \downarrow$

$6678 \downarrow \downarrow$

$55591 \uparrow$

$51776 \uparrow$

$1294 \downarrow$

Figure 3 FH535 inhibited pancreatic cancer cell invasion.

Notes: (A) Dose-dependent inhibition by FH535 of PANC-I and BxPC-3 cell invasion. (B) Microarray analysis of extracellular matrix degradation-related gene expression regulation upon FH535 treatment. (C) Dose-dependent inhibition by FH535 of PANC-I and BxPC-3 cell adhesion. $* P<0.05$, $* * P<0.0 \mathrm{I}$, significant differences vs the respective control groups. (D) Microarray analysis of adhesion molecule-related gene expression regulation upon FH535 treatment. (E) Microarray analysis of EMT-related gene expression regulation upon FH535 treatment. Up and down arrows indicate gene expression significantly upregulated or downregulated, respectively, by twofold. Asterisks indicate genes downstream of the $\mathrm{Wnt} / \mathrm{\beta}$-catenin pathway.

Abbreviation: EMT, epithelial-mesenchymal transition.

but also inhibited cell motility, suggesting the involvement of a similar mechanism.

To establish metastasis, tumor cells must traverse the basement membrane to reach the connective tissues. Accordingly, we investigated the anti-invasive effect of FH535. The Transwell assay proved that FH535 inhibited invasion. In vitro invasion can be divided into several steps, including matrix adhesion, matrix degradation, and EMT. We analyzed the expression of the genes involved in these steps using microarray and found that FH535 significantly downregulated the cell adhesion molecule ITGA5; ITGA5 knockdown results in decreased adhesion in pancreatic cancer cells. ${ }^{48}$ The ability of matrix metalloproteinases (MMPs) to degrade extracellular matrix proteins has been well characterized; therefore, they have been studied extensively to elucidate their involvement in both tumor development and progression. Different MMPs play different roles in tumorigenesis. MMP15 appears to be upregulated during colorectal tumorigenesis, and past research has shown stromal localization of MMP15 in the early phases of neoplastic transformation in colorectal cancer. ${ }^{49}$ In our study, FH535 downregulated MMP15. Epithelial cells are characterized by welldeveloped junctions and apical-basolateral polarization; on the contrary, mesenchymal cells lack polarization due to the loss of an organized junctional layer. Cell metastasis is correlated with EMT. In the present study, FH535 
Table 6 Microarray analysis of extracellular matrix degradationrelated gene expression regulation upon FH535 treatment

\begin{tabular}{llll}
\hline Gene & ID & \multicolumn{2}{l}{ Normalized intensity } \\
\cline { 3 - 4 } & & Control & FH535 \\
\hline TGFBI & 7045 & 16.09069 & 15.894443 \\
TIMP2 & 7077 & 14.796619 & 14.858342 \\
TIMPI & 7076 & 13.919523 & 13.2338505 \\
LAMCI & 3915 & 13.492421 & 14.51922 \\
MMPI & 4312 & 11.905035 & 12.887484 \\
COLI6AI & 1307 & 11.647751 & 10.777789 \\
COL5AI & 1289 & 11.607744 & $10.27258 I$ \\
COL6AI & 1291 & 11.396863 & 10.174638 \\
LAMBI & 3912 & 10.813978 & 9.924841 \\
CTGF & 1490 & 10.809845 & 11.98555 \\
THBS3 & 7059 & 10.1687765 & 9.736564 \\
MMP2 & 4313 & 10.153262 & 9.412593 \\
COL4A2 & 1284 & 9.866227 & 8.850218 \\
THBSI & 7057 & 9.663341 & 9.193558 \\
MMPI5 & 4324 & 9.533566 & 8.395943 \\
SPARC & 6678 & 9.32407 & 8.289816 \\
COL7AI & 1294 & 8.711706 & 7.6560946 \\
LAMB3 & 3914 & 8.550647 & 8.319239 \\
HASI & 3036 & 8.124433 & 7.6573296 \\
ADAMTSI3 & 11093 & 8.074093 & 8.375932 \\
SPG7 & 6687 & 7.799603 & 7.3888316 \\
MMPII & 4320 & 7.460847 & 7.2920337 \\
COLI $2 A I$ & 1303 & 7.404812 & 8.422412 \\
COLI4AI & 7373 & 7.3424816 & 6.805993 \\
TNC & 3371 & 7.329479 & 6.564947 \\
VCAN & 1462 & 7.045421 & 6.763195 \\
MMP9 & 4318 & 7.0101504 & 6.7540355 \\
VTN & 7448 & 6.8925853 & 6.3992944 \\
ECMI & 1893 & 6.8224096 & 7.0186477 \\
ADAMTSI & 9510 & 6.670437 & 7.5566187 \\
CLEC3B & 7123 & 6.6491346 & 6.5587797 \\
SPPI & 6696 & 6.37645 & 6.842924 \\
LAMA3 & 3909 & 6.1783895 & 5.889333 \\
\hline & & &
\end{tabular}

downregulated Snail, which is upregulated during EMT. ${ }^{50}$ In human colorectal cancer cells, overexpression of Snail induces not only EMT but also a cancer stem cell-like phenotype, which enhances cell migration and invasion in vitro and increases metastasis formation in vivo. ${ }^{51}$ Snail also plays an essential role in human pancreatic cancer progression and metastasis. ${ }^{52,53}$ In the clinical setting, overexpression of Snail was previously associated with poorer prognosis and a more invasive phenotype in many malignancies. ${ }^{54-56} \mathrm{We}$ also detected the downregulation of TGFB1, a classic EMT stimulator. ${ }^{57}$ TGFB1 overexpression is associated with early recurrence following resection and decreased survival; ${ }^{58}$ consistent with our study, the suppression of TGFB1 activity in immune-deficient orthotopic mouse models of pancreatic cancer attenuated tumor growth and metastasis. ${ }^{59,60}$

Besides metastasis, FH535 also induced G2/M arrest and inhibited pancreatic cancer cell proliferation. FH535
Table 7 Microarray analysis of adhesion molecule-related gene expression regulation upon $\mathrm{FH} 535$ treatment

\begin{tabular}{|c|c|c|c|}
\hline \multirow[t]{2}{*}{ Gene } & \multirow[t]{2}{*}{ ID } & \multicolumn{2}{|c|}{ Normalized intensity } \\
\hline & & Control & FH535 \\
\hline$C D 44$ & 960 & 15.006962 & 16.199093 \\
\hline ITGB5 & 3693 & | 3.888797 & I 3.484464 \\
\hline LAMCI & 3915 & |3.49242| & 14.51922 \\
\hline$\angle A M B I$ & 3912 & 12.817556 & I3.73472 \\
\hline ITGA3 & 3675 & 12.797894 & $12.39 \mid 577$ \\
\hline ITGBI & 3688 & 12.738785 & 13.636554 \\
\hline ITGAV & 3685 & 12.278682 & 14.3424 \\
\hline ITGA6 & 3655 & 12.273888 & $|4.3924| 8$ \\
\hline ITGA5 & 3678 & 12.169847 & 10.866323 \\
\hline SGCE & 8910 & 12.047686 & $|2.28232|$ \\
\hline ICAMI & 3383 & 12.0056095 & 11.118488 \\
\hline ITGB4 & 3691 & II.982763 & II.56935 \\
\hline CTNNBI & 1499 & II.900537 & 11.841962 \\
\hline CTNNAI & 1495 & 11.841962 & 11.517105 \\
\hline COLI6AI & 1307 & $|1.64775|$ & 10.777789 \\
\hline COL5AI & 1289 & II.607744 & $|0.27258|$ \\
\hline COL6AI & 1291 & II.396863 & 10.174638 \\
\hline CTGF & 1490 & 10.809845 & 11.98555 \\
\hline CTNNDI & 1500 & 10.622252 & 11.350482 \\
\hline THBS3 & 7059 & 10.1687765 & 9.736564 \\
\hline ITGAM & 3684 & 10.003317 & 8.889115 \\
\hline THBSI & 7057 & 9.663341 & 9.193558 \\
\hline ITGA7 & 3679 & 9.627002 & 8.878363 \\
\hline MMPI5 & 4324 & 9.533566 & 8.395943 \\
\hline COL7AI & 1294 & 8.711706 & 7.6560946 \\
\hline LAMB3 & 3914 & 8.550647 & 8.319239 \\
\hline HASI & 3036 & 8.124433 & 7.6573296 \\
\hline ADAMTSI3 & 11093 & 8.074093 & 8.375932 \\
\hline SPG7 & 6687 & $7.9907 / 2$ & 6.850328 \\
\hline COLI2AI & 1303 & $7.4048 \mid 2$ & 8.422412 \\
\hline $\mathrm{CDHI}$ & 999 & 7.3595057 & 7.319695 \\
\hline COLI4AI & 7373 & 7.3424816 & 6.805993 \\
\hline TNC & 3371 & 7.329479 & 6.564947 \\
\hline VCAN & 1462 & 7.045421 & 6.763195 \\
\hline VTN & 7448 & 6.8925853 & 6.3992944 \\
\hline CLEC3B & 7123 & 6.6491346 & 6.5587797 \\
\hline ITGB2 & 3689 & 6.6435785 & $7.7 \mid 3477$ \\
\hline ITGA2 & 3673 & $6.538|4|$ & 10.171594 \\
\hline SPPI & 6696 & 6.37645 & 6.842924 \\
\hline LAMA3 & 3909 & 6.1783895 & 5.889333 \\
\hline SELE & 6401 & 5.8820415 & 7.629673 \\
\hline CTNND2 & $150 \mid$ & 5.4170265 & 5.9111185 \\
\hline ITGAI & 3672 & 5.3547735 & 7.6031985 \\
\hline
\end{tabular}

significantly upregulated the $\mathrm{G} 2 / \mathrm{M}$ regulator gene $B C C I P$ while downregulating the cell cycle regulatory genes $C C N G 1$ and SERTAD1. Human BCCIP, a protein that interacts with BRCA2 and CDKN1A (Cip1, p21), has been implicated in many cellular processes, including cell cycle regulation, DNA recombination and damage repair, telomere maintenance, embryonic development, and genomic stability. ${ }^{61-63}$ 
Table 8 Microarray analysis of EMT-related gene expression regulation upon $\mathrm{FH} 535$ treatment

\begin{tabular}{|c|c|c|c|}
\hline \multirow[t]{2}{*}{ Gene } & \multirow[t]{2}{*}{ ID } & \multicolumn{2}{|c|}{ Normalized intensity } \\
\hline & & Control & FH535 \\
\hline VIM & 7431 & 18.111416 & $18.4 \mid 7988$ \\
\hline TGFBI & 7045 & 16.09069 & 15.894443 \\
\hline NMEI & 4830 & 15.692858 & 15.573043 \\
\hline IGFBP4 & 3487 & $14.852 \mid 57$ & | 3.246835 \\
\hline MSN & 4478 & $|4.75| 84 \mid$ & $15.3576 \mid 6$ \\
\hline RACI & 5879 & 14.251518 & 15.113209 \\
\hline KRT7 & 3855 & 14.184294 & 13.285099 \\
\hline TIMPI & 7076 & 13.919523 & 13.2338505 \\
\hline COL5A2 & 1290 & 13.175857 & 12.833253 \\
\hline TCF3 & 6929 & | 3.00084 & 12.29279 \\
\hline AKTI & 207 & 12.957863 & 12.259176 \\
\hline ITGBI & 3688 & 12.738785 & |3.636554 \\
\hline CAVI & 857 & 12.61244 & 13.617725 \\
\hline SNAII & 6615 & 12.28132 & 10.385736 \\
\hline ITGA5 & 3678 & 12.169847 & 10.866323 \\
\hline TLNI & 7094 & $|2.09664|$ & 10.645829 \\
\hline SYMPK & 8189 & 11.942529 & 12.312602 \\
\hline CTNNBI & 1499 & 11.900537 & II.841962 \\
\hline FXYD5 & 53827 & 11.859393 & 10.980669 \\
\hline BMP7 & 655 & $11.6885 \mid 3$ & $|0.93824|$ \\
\hline COL5AI & 1289 & II.607744 & $|0.27258|$ \\
\hline AHNAK & 79026 & II.605762 & $|0.78592|$ \\
\hline CAV2 & 858 & II.534644 & 11.731664 \\
\hline NOTCH3 & 4854 & II.523583 & 10.370972 \\
\hline TLN2 & 83660 & II.404076 & 10.886059 \\
\hline COL6AI & 1291 & II.396863 & 10.174638 \\
\hline KRTI9 & 3880 & II.289505 & 11.101922 \\
\hline$I G F I R$ & 3480 & II.2369585 & 12.140446 \\
\hline EGFR & 1956 & II.122326 & 12.333595 \\
\hline FYN & 2534 & 10.966112 & II.325876 \\
\hline WASL & 8976 & 10.930079 & 12.483249 \\
\hline CALDI & 800 & 10.921519 & $|2.29469|$ \\
\hline LAMBI & 3912 & 10.813978 & 9.924841 \\
\hline TGFBI & 7040 & 10.7152 & 9.03388 \\
\hline FZD7 & 8324 & 10.711324 & 9.901575 \\
\hline SERPINEI & 5054 & 10.639182 & 10.452353 \\
\hline GRB2 & 2885 & 10.605613 & 9.416897 \\
\hline$R D X$ & 5962 & $|0.52225|$ & 12.191257 \\
\hline SVIL & 6840 & 10.304885 & || $.463 \mid 48$ \\
\hline PLEC & 5339 & 10.301559 & 9.204668 \\
\hline $\mathrm{SHCl}$ & 6464 & 10.287678 & 8.595637 \\
\hline RGS2 & 5997 & 10.257294 & 9.80196 \\
\hline MMP2 & 4313 & 10.153262 & 9.412593 \\
\hline$S S X 2 I P$ & 117178 & 10.144432 & II.167568 \\
\hline COL4A2 & 1284 & 9.866227 & 8.850218 \\
\hline PPAP2B & 8613 & 9.718731 & 9.956581 \\
\hline THBSI & 7057 & 9.663341 & 9.193558 \\
\hline ESAM & 90952 & 9.472261 & 8.959825 \\
\hline NOTCH4 & 4855 & 9.433491 & 9.707824 \\
\hline SPARC & 6678 & 9.32407 & 8.289816 \\
\hline VEZT & 55591 & 9.128493 & ||$.|9545|$ \\
\hline MSTIR & 4486 & 9.085802 & 9.12538 \\
\hline STAT3 & 6774 & 8.847785 & 7.9192953 \\
\hline ZAK & 51776 & 8.719432 & II.337|64 \\
\hline COL7AI & 1294 & 8.711706 & 7.6560946 \\
\hline SMURFI & 57154 & 8.629299 & 9.522539 \\
\hline LAMB3 & 3914 & 8.550647 & 8.319239 \\
\hline TNSI & 7145 & 8.064375 & 7.5303655 \\
\hline
\end{tabular}

(Continued)
Table 8 (Continued)

\begin{tabular}{|c|c|c|c|}
\hline \multirow[t]{2}{*}{ Gene } & \multirow[t]{2}{*}{ ID } & \multicolumn{2}{|c|}{ Normalized intensity } \\
\hline & & Control & FH535 \\
\hline SPG7 & 6687 & 7.990712 & 6.850328 \\
\hline SOSI & 6654 & 7.9648976 & 7.5355105 \\
\hline WIPFI & 7456 & 7.8996034 & 7.0742846 \\
\hline$B M P I$ & 649 & 7.73736 & 6.776738 \\
\hline FOXC2 & 2303 & 7.5557323 & 6.690961 \\
\hline COLI2AI & 1303 & 7.404812 & 8.422412 \\
\hline $\mathrm{CDHI}$ & 999 & 7.3595057 & 7.319695 \\
\hline COLI4AI & 7373 & 7.3424816 & 6.805993 \\
\hline TNC & 3371 & 7.329479 & 6.564947 \\
\hline ILIRN & 3557 & 7.2758436 & 6.734858 \\
\hline SOX 10 & 6663 & 7.0939784 & 6.8492174 \\
\hline VCAN & 1462 & 7.045421 & 6.763195 \\
\hline PTEN & 5728 & 7.0376005 & 9.120998 \\
\hline MMPQ & 4318 & 7.0101504 & 6.7540355 \\
\hline MPP6 & 51678 & 6.9906545 & 8.912582 \\
\hline SYK & 6850 & 6.4246235 & 6.223468 \\
\hline SPPI & 6696 & 6.37645 & 6.842924 \\
\hline$E R B B 3$ & 2065 & 6.178696 & 5.9926624 \\
\hline LAMA3 & 3909 & 6.1783895 & 5.889333 \\
\hline$B M P 2$ & 650 & 6.0141077 & 7.1390386 \\
\hline SOS2 & 6655 & 5.6132765 & 8.797646 \\
\hline TGFB3 & 7043 & 5.5891886 & 6.0552535 \\
\hline
\end{tabular}

Abbreviation: EMT, epithelial-mesenchymal transition.

$B C C I P$ knockdown and concomitant p53 deletion causes rapid development of medulloblastomas, which have a wide spectrum of alterations involving the Sonic hedgehog pathway, consistent with the caretaker responsibility of BCCIP in genomic integrity. ${ }^{64} \mathrm{BCCIP}$ expression is downregulated in human ovarian cancer, renal cell carcinoma, and colorectal cancer tissues, suggesting that the gene plays a role in the pathogenesis of these cancers. ${ }^{63}$ The positive expression rate and intensity of CCNG1 in gastric carcinoma is significantly correlated with tumor differentiation. Elevated amounts of CCNG1 are frequently detected in malignant tissue tumors, including astrocytoma; melanoma; carcinoma of the esophagus, lung, and breast; and cancer of the cervix, uterus, and ovary. ${ }^{65}$ It plays a pivotal role in hepatocellular carcinoma metastasis and may be a novel prognostic biomarker and therapeutic target. ${ }^{66}$ SERTAD1 is involved in positive regulation of the cell cycle and proliferation; ${ }^{67,68}$ accordingly, its expression is upregulated in several tumor types. ${ }^{69,70}$ Studies indicate that SERTAD1 promotes proliferation by binding to the transcription factor E2F1 and by enhancing its transcriptional activity. ${ }^{71}$ Experimental overexpression of SERTAD1 provoked hyperproliferation, ${ }^{72}$ genomic instability ${ }^{68}$ and inhibition of apoptosis. ${ }^{73}$

We demonstrated that FH535 significantly inhibits pancreatic cancer cell metastasis by suppressing migration, invasion, and adhesion and induces the accumulation of cells in the $\mathrm{G} 2 / \mathrm{M}$ phase to suppress proliferation. These results 
suggest that FH535 is a potential candidate for pancreatic cancer treatment. Some of the identified genes that responded to FH535 are well-established direct targets of the Wnt/ $\beta$-catenin pathway. However, it has not been proven that the other identified genes are located downstream of the pathway. FH535 might affect the expression of these genes through the $\mathrm{Wnt} / \beta$-catenin pathway indirectly or in

A
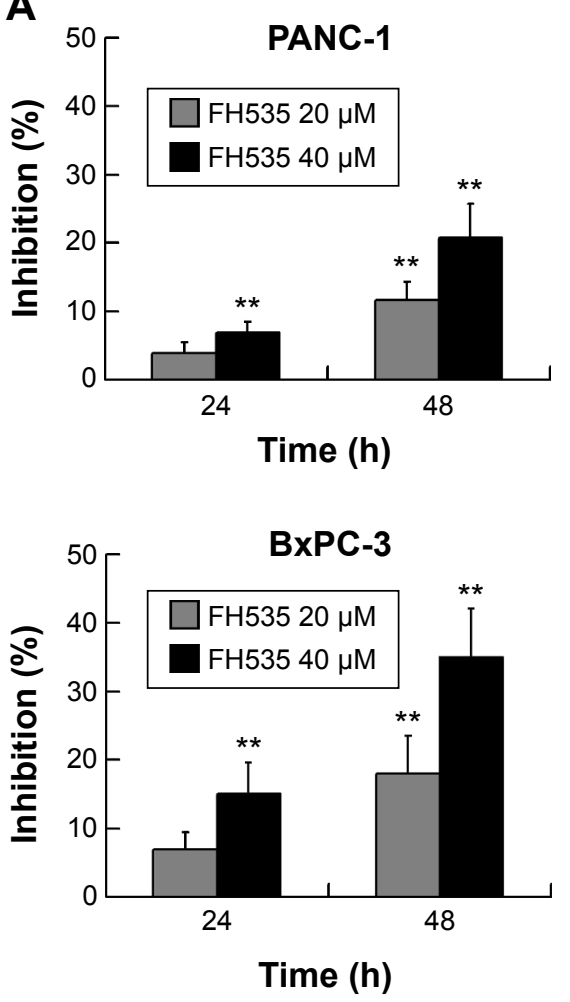

B
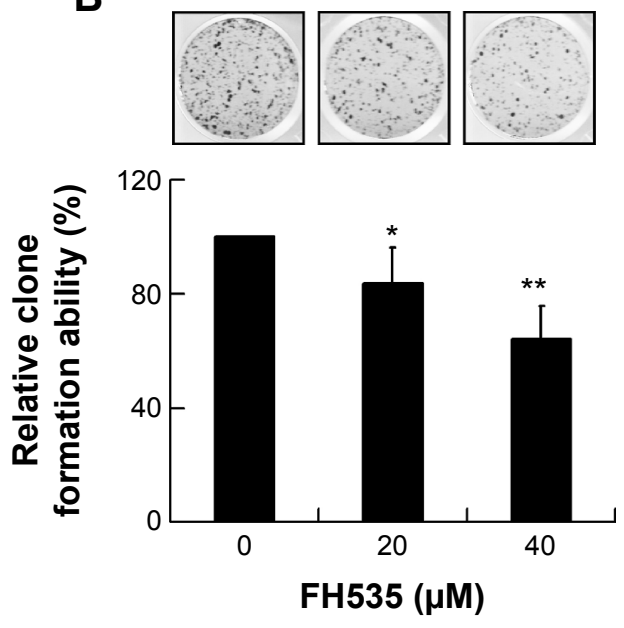

a $\beta$-catenin independent manner. In fact, FH535 not only antagonizes $\beta$-catenin/TCF-mediated transcription but also inhibits recruitment of the coactivators glucocorticoid receptor-interacting protein 1 (GRIP1) and $\beta$-catenin to peroxisome proliferator-activated receptor (PPAR) $\delta$ and PPAR $\gamma,{ }^{10}$ suggesting that these mechanisms could also be involved in the anti-cancer effect of FH535.
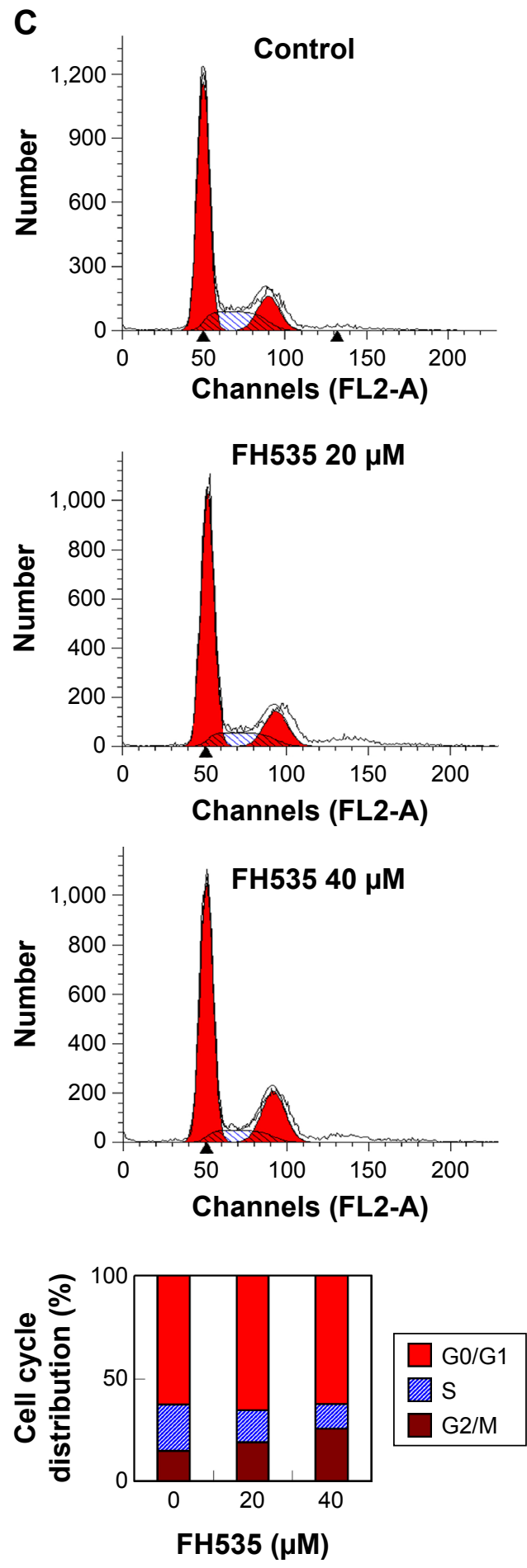


\section{D}
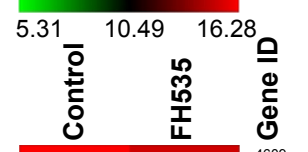

$G^{e^{n}}$

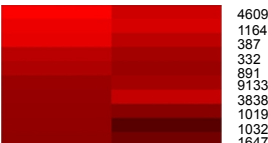

MYC

KPNA2

CDKN2D

CDKN1C

$D D I T 3$

SERTAD 1

GNL3

RASSF1

$C D C 34$

$C D C 20$

CUL3

FOSL1

$\mathrm{FOXO} 3$

$P D K 2$

WEE1

$A U R K B$

PPP1R15A

CCND3

RAD1

EGFR

$C D C 16$

$B C C I P$

RB1

GRB2

PPM1D

PKD1

E2F3

CCNG1

MAD2L2

$\mathrm{SHC1}$

CCNT1

RUVBL1

SKP2

$A H R$

CCNG2

RBBP8

MAPK3

CDK5RAP2

MKI67

CUL1

RBL2

E2F4

PTK2

CDK5RAP1

ATR

CDKN1A

CDKN1B

TSC1

BRCA1

CUL2

PTEN

MDM4

RBL1

BMP2

SOX2

PTGS2

APC

BTRC
$D$

4609

3838

1032

1028

1649

29950

26354

11186

997

991

8452

8061

2309

5164

7465

9212

23645

896

5810

1956

8881

56647

5925

2885

8493

5310

1871

900

10459

6464

904

8607

6502

196

901

5932

5595

55755

4288

8454

5934

1874

5747

51654

545

1026

1027

7248

672

8453

5728

4194

5933

650

6657

5743

324

8945

Table 9 Microarray analysis of cell cycle-related gene expression regulation upon $20 \mu \mathrm{M}$ FH535 treatment

\begin{tabular}{|c|c|c|c|}
\hline \multirow[t]{2}{*}{ Gene } & \multirow[t]{2}{*}{ ID } & \multicolumn{2}{|c|}{ Normalized intensity } \\
\hline & & Control & FH535 \\
\hline MYC & 4609 & 16.268158 & 15.204586 \\
\hline CKS2 & 1164 & $15.878 \mid 64$ & |5.39457| \\
\hline RHOA & 387 & $15.76 \mid 177$ & $|4.78665|$ \\
\hline BIRC5 & 332 & 14.757564 & 14.219355 \\
\hline CCNBI & 891 & |4.478785 & 14.022737 \\
\hline CCNB2 & 9133 & |4.0|987| & $14.27 \mid 269$ \\
\hline KPNA2 & 3838 & $13.950 \mid 85$ & $|4.97| 469$ \\
\hline CDK4 & 1019 & 13.87332 & 13.635977 \\
\hline CDKN2D & 1032 & 13.839806 & 12.513427 \\
\hline GADD45A & 1647 & $|3.76545|$ & 13.130958 \\
\hline CDKNIC & 1028 & 13.694702 & $12.537 \mid 76$ \\
\hline PCNA & 5111 & $13.6 \mid 1973$ & $|3.24| 4665$ \\
\hline CKSIB & 1163 & 13.609195 & 12.845673 \\
\hline MCM3 & 4172 & | 3.580797 & $13.9|195|$ \\
\hline PRCI & 9055 & 13.4|| 4275 & |4.3247| \\
\hline MAD2LI & 4085 & I 3.297964 & 12.867024 \\
\hline CDKI & 983 & 13.286596 & 13.600226 \\
\hline DDIT3 & 1649 & 13.135856 & 11.871853 \\
\hline SERTAD I & 29950 & | 3.060848 & 11.212 \\
\hline IGF2 & 3481 & | 3.0203 & $13.7997 \mid 7$ \\
\hline AKTI & 207 & 12.957863 & 12.259176 \\
\hline GNL3 & 26354 & $12.89 \mid 848$ & 14.223899 \\
\hline ITGBI & 3688 & 12.738785 & 13.636554 \\
\hline RASSFI & 11186 & 12.559567 & II.458946 \\
\hline CDK7 & 1022 & 12.553116 & |3.288865 \\
\hline CDC34 & 997 & 12.53036 & II.302476 \\
\hline TFDP2 & 7029 & 12.527303 & 12.6644745 \\
\hline$C D C 20$ & 991 & 12.526335 & II.232994 \\
\hline CDKN3 & 1033 & 12.486179 & 12.966997 \\
\hline MAP2KI & 5604 & I 2.483637 & 11.714967 \\
\hline CUL3 & 8452 & 12.389215 & 13.715795 \\
\hline FOSLI & 8061 & 12.344017 & II.102832 \\
\hline ILK & 3611 & 12.11682 & II.583433 \\
\hline FOXO3 & 2309 & $|2.0950| \mid$ & I3.865094 \\
\hline MNATI & 4331 & I 2.074865 & 12.456777 \\
\hline JUN & 3725 & 12.038464 & 12.673436 \\
\hline PDK2 & 5164 & 11.944916 & $10.78 \mid 894$ \\
\hline CTNNBI & 1499 & 11.900537 & 11.841962 \\
\hline WEEI & 7465 & 11.894105 & $13.38 \mid 722$ \\
\hline MCM2 & $4|7|$ & | | & II.269842 \\
\hline AURKB & 9212 & II.800928 & 9.697033 \\
\hline PPPIRI5A & 23645 & 11.676006 & 10.124018 \\
\hline $\mathrm{MDCl}$ & 9656 & 11.649198 & $10.9406 \mid 4$ \\
\hline CDKN2C & $103 \mid$ & 11.6150875 & II.096327 \\
\hline CCND3 & 896 & I I.576098 & 10.075832 \\
\hline CCNEI & 898 & 11.407219 & II.045229 \\
\hline CDKN2A & 1029 & II.343829 & II.097562 \\
\hline AURKA & 6790 & 11.181647 & 10.961699 \\
\hline RADI & 5810 & 11.162613 & 9.890079 \\
\hline $\mathrm{NOTCH} 2$ & 4853 & II.125797 & 10.202223 \\
\hline EGFR & 1956 & II.122326 & 12.333595 \\
\hline CDK5 & 1020 & 11.117936 & 10.542482 \\
\hline RAD5I & 5888 & 11.1082325 & 10.784544 \\
\hline CCNA2 & 890 & $10.9|186|$ & 10.9871645 \\
\hline MCM4 & 4173 & 10.849212 & II.05679 \\
\hline $\mathrm{CDCl} 6$ & 8881 & 10.808938 & 13.084003 \\
\hline
\end{tabular}

(Continued)

Figure 4 Inhibitory effect of FH535 on pancreatic cancer cell growth.

Notes: (A) Dose- and time-dependent inhibition by FH535 of PANC-I and BxPC-3 cell growth. (B) Dose-dependent inhibition by $\mathrm{FH} 535$ of the clone formation ability of BxPC-3 cells. $* P<0.05, * * P<0.01$, significant differences vs the respective control groups. (C) Significant dose-dependent G2/M arrest following FH535 treatment in BxPC-3 cells. (D) Microarray analysis of cell cycle-related gene expression regulation upon $20 \mu \mathrm{M}$ FH535 treatment. Up and down arrows indicate gene expression significantly upregulated or downregulated, respectively, by twofold. Asterisks indicate genes downstream of the $\mathrm{Wnt} / \beta$-catenin pathway. Abbreviation: h, hours. 
Table 9 (Continued)

\begin{tabular}{|c|c|c|c|}
\hline \multirow[t]{2}{*}{ Gene } & \multirow[t]{2}{*}{ ID } & \multicolumn{2}{|c|}{ Normalized intensity } \\
\hline & & Control & FH535 \\
\hline BCCIP & 56647 & $|0.778| 8 \mid$ & 12.021907 \\
\hline$C D C 25 C$ & 995 & 10.672214 & 10.564099 \\
\hline$R B I$ & 5925 & 10.640414 & 12.741512 \\
\hline CCNC & 892 & 10.610059 & 10.50804 \\
\hline CDK9 & 1025 & 10.606858 & 9.6394415 \\
\hline GRB2 & 2885 & 10.605613 & 9.416897 \\
\hline ATM & 472 & 10.5960865 & 9.790577 \\
\hline PPMID & 8493 & 10.580978 & 9.338833 \\
\hline CCNDI & 595 & $10.54362 \mid$ & 10.14267 \\
\hline PKDI & 5310 & 10.471296 & 9.29677 \\
\hline E2F3 & |87| & 10.412796 & 12.061844 \\
\hline CCNGI & 900 & $10.40930 \mid$ & 9.063653 \\
\hline CDK5RI & 8851 & 10.322197 & 10.292204 \\
\hline PDKI & 5163 & 10.321034 & 10.933424 \\
\hline$M A D 2 L 2$ & 10459 & 10.306548 & 9.040705 \\
\hline $\mathrm{SHCl}$ & 6464 & 10.287678 & 8.595637 \\
\hline CCNTI & 904 & 10.17827 & 11.456285 \\
\hline CDK2 & 1017 & 10.10528 & 9.357359 \\
\hline RUVBLI & 8607 & 10.000026 & 8.814938 \\
\hline SKP2 & 6502 & 9.937107 & 8.872935 \\
\hline CASP3 & 836 & 9.893856 & 10.658698 \\
\hline$A H R$ & 196 & 9.886938 & |I.93648| \\
\hline RAD9A & 5883 & 9.855825 & 8.961951 \\
\hline PA2G4 & 5036 & 9.737894 & 8.966842 \\
\hline CHEK2 & 11200 & 9.726725 & 10.255492 \\
\hline INS & 3630 & 9.673779 & 8.813154 \\
\hline BBS4 & 585 & 9.597437 & 9.65494 \\
\hline SIAHI & 6477 & 9.530121 & 9.295226 \\
\hline CCNG2 & 901 & 9.4692955 & 8.337656 \\
\hline RBBP8 & 5932 & 9.454372 & 12.631638 \\
\hline MAPK3 & 5595 & 9.447224 & 7.818405 \\
\hline TFDPI & 7027 & 9.4434185 & 9.030092 \\
\hline $\mathrm{E} 2 \mathrm{FI}$ & 1869 & $9.397 \mid 23$ & 8.754342 \\
\hline CDK5RAP2 & 55755 & 9.390803 & 10.79269 \\
\hline MAPKI & 5594 & 9.240688 & 9.218932 \\
\hline MKI67 & 4288 & 9.233824 & 8.092867 \\
\hline ID2 & 3398 & 9.226584 & 8.999163 \\
\hline CULI & 8454 & 9.221058 & 10.794849 \\
\hline$R B L 2$ & 5934 & 9.160267 & 10.348637 \\
\hline JAG2 & 3714 & 9.075178 & 8.140677 \\
\hline GTSEI & 51512 & 9.04477 & 8.488665 \\
\hline CHEKI & 1111 & 9.04231 & 9.651725 \\
\hline$E 2 F 4$ & 1874 & 9.035055 & 8.016477 \\
\hline GOS2 & 50486 & 9.032754 & 8.410861 \\
\hline SESN2 & 83667 & 8.951754 & 8.138044 \\
\hline PTK2 & 5747 & 8.939062 & 10.820772 \\
\hline$C D C 25 A$ & 993 & 8.677163 & 8.042739 \\
\hline MDM2 & 4193 & 8.622698 & 7.73673 \\
\hline CDK5RAPI & 51654 & 8.508385 & 6.9220624 \\
\hline AXINI & 8312 & 8.351635 & 7.6164603 \\
\hline ATR & 545 & 8.152882 & 11.634625 \\
\hline MCM5 & 4174 & 8.055058 & 7.4200873 \\
\hline CDKNIA & 1026 & 8.007444 & 6.7135 \\
\hline SOSI & 6654 & 7.9648976 & 7.5355105 \\
\hline CDKNIB & 1027 & 7.8906517 & 6.7354736 \\
\hline GML & 2765 & 7.8596773 & 6.9595275 \\
\hline
\end{tabular}

Table 9 (Continued)

\begin{tabular}{llll}
\hline Gene & ID & \multicolumn{2}{l}{ Normalized intensity } \\
\cline { 3 - 4 } & & Control & FH535 \\
\hline TSCI & 7248 & 7.6562896 & 9.700143 \\
BRCAI & 672 & 7.620017 & 10.24544 \\
CUL2 & 8453 & 7.566332 & 9.5496025 \\
STMNI & 3925 & 7.5155845 & 6.8102884 \\
CDK8 & 1024 & 7.5133963 & 6.9160185 \\
TERT & 7015 & 7.4070444 & 7.4882307 \\
ABLI & 25 & 7.3099413 & 6.6226487 \\
PTEN & 5728 & 7.0376005 & 9.120998 \\
MDM4 & 4194 & 6.996299 & 8.63818 \\
HUSI & 3364 & 6.976554 & 7.3689637 \\
RBLI & 5933 & 6.7265186 & 8.035306 \\
CCND2 & 894 & 6.3239446 & 5.996339 \\
BMP2 & 650 & 6.0141077 & 7.1390386 \\
ATRIP & 84126 & 5.912352 & 6.682503 \\
CDC6 & 990 & 5.8019896 & 6.064807 \\
SOX2 & 6657 & 5.6166873 & 6.7975965 \\
PTGS2 & 5743 & 5.5262737 & 7.601541 \\
APC & 324 & 5.3153567 & 6.5241365 \\
BTRC & 8945 & 5.3152456 & 7.7473273 \\
\hline
\end{tabular}

\section{Acknowledgments}

This study was supported by the National Natural Science Foundation of China (grant nos 81472296, 81101867, 81272542, 81200369, and 81372443), the CSPAC-Celgene Foundation, the China International Medical Foundation (grant no CIMF-F-H001-057), the Special Foundation of Clinical Medicine of Jiangsu Provincial Bureau of Science and Technology (grant no BL2014039), the Scientific Research Project of Jiangsu Provincial Bureau of Traditional Chinese Medicine (grant no L213236), the Medical Scientific Research Project of Jiangsu Provincial Bureau of Health (grant no Z201206), the Special Foundation of Wu Jieping Medical Foundation for Clinical Scientific Research (grant nos 320.6753.1225 and 320.6750.12242), the Science and Education for Health Foundation of Suzhou for Youth (grant nos SWKQ1003 and SWKQ1011), the Science and Technology Project Foundation of Suzhou (grant nos SYS201112, SYSD2012137, and SYS201335), the Science and Technology Foundation of Suzhou Xiangcheng (grant nos SZXC2012-70 and XJ201451), and a project founded by the priority academic program development of Jiangsu higher education institutions.

\section{Disclosure}

The authors report no conflicts of interest in this work.

\section{References}

1. Jemal A, Siegel R, Ward E, et al. Cancer statistics, 2006. CA Cancer J Clin. 2006;56(2):106-130.

2. Li D, Xie K, Wolff R, Abbruzzese JL. Pancreatic cancer. Lancet. 2004;363(9414):1049-1057. 
3. Bosetti C, Bertuccio P, Malvezzi M, et al. Cancer mortality in Europe, 2005-2009, and an overview of trends since 1980. Ann Oncol. 2013; 24(10):2657-2671.

4. Cai HH, Sun YM, Miao Y, et al. Aberrant methylation frequency of TNFRSF10C promoter in pancreatic cancer cell lines. Hepatobiliary Pancreat Dis Int. 2011;10(1):95-100.

5. Iida J, Wilhelmson KL, Price MA, et al. Membrane type-1 matrix metalloproteinase promotes human melanoma invasion and growth. J Invest Dermatol. 2004;122(1):167-176.

6. Eisenmann KM, McCarthy JB, Simpson MA, et al. Melanoma chondroitin sulphate proteoglycan regulates cell spreading through $\mathrm{Cdc} 42$, Ack-1 and p130cas. Nat Cell Biol. 1999;1(8):507-513.

7. Vaid M, Prasad R, Sun Q, Katiyar SK. Silymarin targets beta-catenin signaling in blocking migration/invasion of human melanoma cells. PLoS One. 2011;6(7):e23000.

8. Iida J, Pei D, Kang T, et al. Melanoma chondroitin sulfate proteoglycan regulates matrix metalloproteinase-dependent human melanoma invasion into type I collagen. J Biol Chem. 2001;276(22): 18786-18794.

9. Clevers H. Wnt/beta-catenin signaling in development and disease. Cell. 2006;127(3):469-480.

10. Handeli S, Simon JA. A small-molecule inhibitor of Tcf/beta-catenin signaling down-regulates PPARgamma and PPARdelta activities. $\mathrm{Mol}$ Cancer Ther. 2008;7(3):521-529.

11. Iida J, Dorchak J, Lehman JR, et al. FH535 inhibited migration and growth of breast cancer cells. PLoS One. 2012;7(9):e44418.

12. Ren J, Wang R, Song H, Huang G, Chen L. Secreted frizzled related protein 1 modulates taxane resistance of human lung adenocarcinoma. Mol Med. 2014;20:164-178.

13. Hannigan G, Troussard AA, Dedhar S. Integrin-linked kinase: a cancer therapeutic target unique among its ILK. Nat Rev Cancer. 2005; 5(1):51-63.

14. Legate KR, Montanez E, Kudlacek O, Fassler R. ILK, PINCH and parvin: the tIPP of integrin signalling. Nat Rev Mol Cell Biol. 2006;7(1): 20-31.

15. Palacios F, Price L, Schweitzer J, Collard JG, D’Souza-Schorey C. An essential role for ARF6-regulated membrane traffic in adherens junction turnover and epithelial cell migration. EMBOJ. 2001;20(17): 4973-4986.

16. Pece S, Gutkind JS. E-cadherin and Hakai: signalling, remodeling or destruction? Nat Cell Biol. 2002;4(4):E72-E74.

17. D'Souza-Schorey C. Disassembling adherens junctions: breaking up is hard to do. Trends Cell Biol. 2005;15(1):19-26.

18. Tsukita S, Furuse M, Itoh M. Multifunctional strands in tight junctions. Nat Rev Mol Cell Biol. 2001;2(4):285-293.

19. Cheng CY, Mruk DD. Cell junction dynamics in the testis: sertoli-germ cell interactions and male contraceptive development. Physiol Rev. 2002;82(4):825-874.

20. Matter K, Balda MS. Signalling to and from tight junctions. Nat Rev Mol Cell Biol. 2003;4(3):225-236.

21. Balda MS, Matter K. Epithelial cell adhesion and the regulation of gene expression. Trends Cell Biol. 2003;13(6):310-318.

22. Bazzoni G, Dejana E. Endothelial cell-to-cell junctions: molecular organization and role in vascular homeostasis. Physiol Rev. 2004;84(3): 869-901.

23. Furuse M, Tsukita S. Claudins in occluding junctions of humans and flies. Trends Cell Biol. 2006;16(4):181-188.

24. Linder S. The matrix corroded: podosomes and invadopodia in extracellular matrix degradation. Trends Cell Biol. 2007;17(3): $107-117$.

25. Chhabra ES, Higgs HN. The many faces of actin: matching assembly factors with cellular structures. Nat Cell Biol. 2007;9(10):1110-1121.

26. McEver RP, Zhu C. Rolling cell adhesion. Annu Rev Cell Dev Biol. 2010;26:363-396.

27. Parsons JT, Horwitz AR, Schwartz MA. Cell adhesion: integrating cytoskeletal dynamics and cellular tension. Nat Rev Mol Cell Biol. 2010; 11(9):633-643.
28. Mullins RF, Skeie JM, Folk JC, et al. Evaluation of variants in the selectin genes in age-related macular degeneration. BMC Med Genet. 2011;12:58.

29. Ciriza J, Garcia-Ojeda ME. Expression of migration-related genes is progressively upregulated in murine lineage-Sca-1+c-Kit+ population from the fetal to adult stages of development. Stem Cell Res Ther. 2010; $1(2): 14$.

30. Kaartinen V, Voncken JW, Shuler C, et al. Abnormal lung development and cleft palate in mice lacking TGF-beta 3 indicates defects of epithelial-mesenchymal interaction. Nat Genet. 1995;11(4):415-421.

31. Timmerman LA, Grego-Bessa J, Raya A, et al. Notch promotes epithelial-mesenchymal transition during cardiac development and oncogenic transformation. Genes Dev. 2004;18(1):99-115.

32. Moreno-Bueno G, Cubillo E, Sarrió D, et al. Genetic profiling of epithelial cells expressing E-cadherin repressors reveals a distinct role for snail, slug, and E47 factors in epithelial-mesenchymal transition. Cancer Res. 2006;66(19):9543-9556.

33. Yang J, Weinberg RA. Epithelial-mesenchymal transition: at the crossroads of development and tumor metastasis. Dev Cell. 2008;14(6): $818-829$.

34. Hoffman AE, Zheng T, Ba Y, et al. Phenotypic effects of the circadian gene cryptochrome 2 on cancer-related pathways. BMC Cancer. 2010;10:110.

35. Tetsu O, McCormick F. Beta-catenin regulates expression of cyclin D1 in colon carcinoma cells. Nature. 1999;398(6726):422-426.

36. Fearon ER. PARsing the phrase "all in for axin" - Wnt pathway targets in cancer. Cancer Cell. 2009;16(5):366-368.

37. White BD, Chien AJ, Dawson DW. Dysregulation of Wnt/beta-catenin signaling in gastrointestinal cancers. Gastroenterology. 2012;142(2): 219-232.

38. MacDonald BT, Tamai K, He X. Wnt/beta-catenin signaling: components, mechanisms, and diseases. Dev Cell. 2009;17(1):9-26.

39. Di Cristofano A, Pandolfi PP. The multiple roles of PTEN in tumor suppression. Cell. 2000;100(4):387-390.

40. Waite KA, Eng C. Protean PTEN: form and function. Am J Hum Genet. 2002;70(4):829-844.

41. Chalhoub N, Baker SJ. PTEN and the PI3-kinase pathway in cancer. Annu Rev Pathol. 2009;4:127-150.

42. Wang H, Chen P, Liu XX, et al. Prognostic impact of gastrointestinal bleeding and expression of PTEN and Ki-67 on primary gastrointestinal stromal tumors. World J Surg Oncol. 2014;12:89.

43. Tang H, Yao L, Tao X, et al. miR-9 functions as a tumor suppressor in ovarian serous carcinoma by targeting TLN1. Int J Mol Med. 2013; 32(2):381-388.

44. Sakamoto S, McCann RO, Dhir R, Kyprianou N. Talin1 promotes tumor invasion and metastasis via focal adhesion signaling and anoikis resistance. Cancer Res. 2010;70(5):1885-1895.

45. Sommers CL, Byers SW, Thompson EW, Torri JA, Gelmann EP. Differentiation state and invasiveness of human breast cancer cell lines. Breast Cancer Res Treat. 1994;31(2-3):325-335.

46. Zhu H, Lu J, Wang X, et al. Upregulated ZO-1 correlates with favorable survival of gastrointestinal stromal tumor. Med Oncol. 2013;30(3): 631.

47. Doi Y, Yashiro M, Yamada N, Amano R, Noda S, Hirakawa K. VEGF-A/ VEGFR-2 signaling plays an important role for the motility of pancreas cancer cells. Ann Surg Oncol. 2012;19(8):2733-2743.

48. Walsh N, Clynes M, Crown J, O'Donovan N. Alterations in integrin expression modulates invasion of pancreatic cancer cells. J Exp Clin Cancer Res. 2009;28:140.

49. Sena P, Mariani F, Marzona L, et al. Matrix metalloproteinases 15 and 19 are stromal regulators of colorectal cancer development from the early stages. Int J Oncol. 2012;41(1):260-266.

50. Wu Y, Zhou BP. Snail: more than EMT. Cell Adh Migr. 2010;4(2): 199-203.

51. Fan F, Samuel S, Evans KW, et al. Overexpression of snail induces epithelial-mesenchymal transition and a cancer stem cell-like phenotype in human colorectal cancer cells. Cancer Med. 2012;1(1):5-16. 
52. Hotz B, Arndt M, Dullat S, Bhargava S, Buhr HJ, Hotz HG. Epithelial to mesenchymal transition: expression of the regulators snail, slug, and twist in pancreatic cancer. Clin Cancer Res. 2007;13(16):4769-4776.

53. von Burstin J, Eser S, Paul MC, et al. E-cadherin regulates metastasis of pancreatic cancer in vivo and is suppressed by a SNAIL/HDAC1/HDAC2 repressor complex. Gastroenterology. 2009;137(1):e361-e365.

54. Shin NR, Jeong EH, Choi CI, et al. Overexpression of snail is associated with lymph node metastasis and poor prognosis in patients with gastric cancer. BMC Cancer. 2012;12:521.

55. Kuo KT, Chou TY, Hsu HS, Chen WL, Wang LS. Prognostic significance of NBS1 and snail expression in esophageal squamous cell carcinoma. Ann Surg Oncol. 2012;19(suppl 3):S549-S557.

56. van Nes JG, de Kruijf EM, Putter H, et al. Co-expression of SNAIL and TWIST determines prognosis in estrogen receptor-positive early breast cancer patients. Breast Cancer Res Treat. 2012;133(1):49-59.

57. Wu Y, Zhou BP. New insights of epithelial-mesenchymal transition in cancer metastasis. Chin J Biochem Biophys. 2008;40(7):643-650.

58. Friess H, Yamanaka Y, Büchler M, et al. Enhanced expression of transforming growth factor beta isoforms in pancreatic cancer correlates with decreased survival. Gastroenterology. 1993;105(6):1846-1856.

59. Rowland-Goldsmith MA, Maruyama H, Kusama T, Ralli S, Korc M. Soluble type II transforming growth factor-beta (TGF-beta) receptor inhibits TGF-beta signaling in COLO-357 pancreatic cancer cells in vitro and attenuates tumor formation. Clin Cancer Res. 2001;7(9): 2931-2940.

60. Melisi D, Ishiyama S, Sclabas GM, et al. LY2109761, a novel transforming growth factor beta receptor type I and type II dual inhibitor, as a therapeutic approach to suppressing pancreatic cancer metastasis. Mol Cancer Ther. 2008;7(4):829-840.

61. Misra S, Sharma S, Agarwal A, et al. Cell cycle-dependent regulation of the bi-directional overlapping promoter of human BRCA2/ZAR2 genes in breast cancer cells. Mol Cancer. 2010;9:50.

62. Lu H, Yue J, Meng X, Nickoloff JA, Shen Z. BCCIP regulates homologous recombination by distinct domains and suppresses spontaneous DNA damage. Nucleic Acids Res. 2007;35(21):7160-7170.

63. Liu X, Cao L, Ni J, et al. Differential BCCIP gene expression in primary human ovarian cancer, renal cell carcinoma and colorectal cancer tissues. Int J Oncol. 2013;43(6):1925-1934.
64. Huang YY, Dai L, Gaines D, et al. BCCIP suppresses tumor initiation but is required for tumor progression. Cancer Res. 2013;73(23): $7122-7133$

65. Cui X, Yu L, Wang Y, et al. The relationship between cyclin G1 and survival in patients treated surgically for HCC. Hepatogastroenterology. 2013;60(121):153-159.

66. Wen W, Ding J, Sun W, et al. Cyclin G1-mediated epithelial-mesenchymal transition via phosphoinositide 3-kinase/Akt signaling facilitates liver cancer progression. Hepatology. 2012;55(6):1787-1798.

67. Li J, Muscarella P, Joo SH, et al. Dissection of CDK4-binding and transactivation activities of p34(SEI-1) and comparison between functions of p34(SEI-1) and p16(INK4A). Biochemistry. 2005;44(40): 13246-13256.

68. Tang DJ, Hu L, Xie D, et al. Oncogenic transformation by SEI-1 is associated with chromosomal instability. Cancer Res. 2005;65(15): 6504-6508.

69. van Dekken H, Alers JC, Riegman PH, Rosenberg C, Tilanus HW, Vissers K. Molecular cytogenetic evaluation of gastric cardia adenocarcinoma and precursor lesions. Am J Pathol. 2001;158(6):1961-1967.

70. Tang TC, Sham JS, Xie D, et al. Identification of a candidate oncogene SEI-1 within a minimal amplified region at $19 \mathrm{q} 13.1$ in ovarian cancer cell lines. Cancer Res. 2002;62(24):7157-7161.

71. Hsu SI, Yang CM, Sim KG, Hentschel DM, O'Leary E, Bonventre JV. TRIP-Br: a novel family of PHD zinc finger- and bromodomaininteracting proteins that regulate the transcriptional activity of E2F-1/ DP-1. EMBO J. 2001;20(9):2273-2285.

72. Sugimoto M, Nakamura T, Ohtani N, et al. Regulation of CDK4 activity by a novel CDK4-binding protein, p34(SEI-1). Genes Dev. 1999; 13(22):3027-3033

73. Hong SW, Kim CJ, Park WS, et al. p34SEI-1 inhibits apoptosis through the stabilization of the X-linked inhibitor of apoptosis protein: p34SEI-1 as a novel target for anti-breast cancer strategies. Cancer Res. 2009;69(3):741-746
OncoTargets and Therapy

\section{Publish your work in this journal}

OncoTargets and Therapy is an international, peer-reviewed, open access journal focusing on the pathological basis of all cancers, potential targets for therapy and treatment protocols employed to improve the management of cancer patients. The journal also focuses on the impact of management programs and new therapeutic agents and protocols on

\section{Dovepress}

patient perspectives such as quality of life, adherence and satisfaction The manuscript management system is completely online and includes a very quick and fair peer-review system, which is all easy to use. Visit http://www.dovepress.com/testimonials.php to read real quotes from published authors. 